\title{
Principles and Applications of SQUIDs
}

\author{
JOHN CLARKE
}

Invited Paper

Superconducting quantum interference devices (SQUIDs) combine flux quantization and Josephson tunneling to produce an output voltage that is periodic in the applied magnetic flux with a period of one flux quantum, $\Phi_{0}$. SQUIDs are thus flux-to-voltage transducers, and are able to detect changes in flux much smaller than $\Phi_{0}$. Using Nb-based thin-film technology, a number of groups can now routinely fabricate dc SQUIDs with input circuits integrated on the same chip. At $4.2 \mathrm{~K}$ the dc SQUID, which operates with a constant bias current, can detect signals with an equivalent energy per unit bandwidth of a few hundred h. At low frequencies, the SQUID exhibits $1 / \mathrm{f}$ noise that originates in fluctuations in the critical current of the iunctions and in the motion of magnetic flux trapped in the thin films. RF SQUIDs, which operate with an RF flux bias, are usually machined from bulk $\mathrm{Nb}$, and have a typical energy sensitivity per unit bandwidth of $10^{5}$ to $10^{6} \mathrm{~h}$. Both types of SQUID can be used in a wide variety of applications, including magnetometers, gradiometers, susceptometers, voltmeters, $R F$ amplifiers, gravity wave antennas, and gravity gradiometers. Both dc and RF SQUIDs have been fabricated from high-temperature superconductors and operated at temperatures of up to $77 \mathrm{~K}$. These devices exhibit high levels of $1 / \mathrm{f}$ noise, which generally arises from the motion of trapped magnetic flux.

\section{INTRODUCTION}

The superconducting quantum interference devices (SQUIDs) are the most sensitive detectors of magnetic flux available. A SQUID is, in essence, a flux-to-voltage transducer, providing an output voltage that is periodic in the applied flux with a period of one flux quantum, $\Phi_{0} \equiv h / 2 \mathrm{e}$ $\approx 2.07 \times 10^{-15} \mathrm{~Wb}$. One is generally able to detect an output signal corresponding to a flux change of much less than $\Phi_{0}$. SQUIDs are amazingly versatile, being able to measure any physical quantity that can be converted to a flux, for example, magnetic field, magnetic field gradient, current, voltage, displacement, and magnetic susceptibility. As a result, their applications are wide ranging, from the detection of tiny magnetic fields produced by the human brain and the measurement of fluctuating magnetic fields in remote areas to the detection of gravity waves and the observation of spin noise in an ensemble of magnetic nuclei.

Manuscript received October 27, 1988; revised April 14, 1989. This work was supported by the U.S. Department of Energy under Contract DE-AC03-76SF00098. This paper is a shortened version of a chapter in Proceedings of the NATO Advanced Study Institute on Superconductive Electronics, M. Nisenoff and $\mathrm{H}$. Weinstock, Eds.

The author is with the Department of Physics, University of California, and the Materials and Chemical Sciences Division, Lawrence Berkeley Laboratory, Berkeley, CA 94720, USA.

IEEE Log Number 8929376.
SQUIDs combine two physical phenomena, flux quantization-the fact that the flux $\Phi$ in a closed superconducting loop is quantized [1] in units of $\Phi_{0}$-and Josephson tunneling [2]. There are two kinds of SQUIDs. The first [3], the dc SQUID, consists of two Josephson junctions connected in parallel on a superconducting loop and is so named because it operates with a steady current bias. Relatively crude devices were developed in the second half of the 1960 s and used successfully by low-temperature physicists to measure a variety of phenomena occurring at liquid helium temperatures. At the end of the decade, the radio frequency (RF) SQUID [4], [5] appeared. This device involves a single Josephson junction, interrupting the current flow around a superconducting loop, and is operated with an RF flux bias.

Because it required only a single junction, at the time the RF SQUID was simpler to manufacture and quickly became available commercially. It has remained the more widely used device ever since. However, in the mid-1970s it was shown that the dc SQUID was the more sensitive device, and there has been an ongoing development of thin-film dc SQUIDs and instruments based on them since then. By contrast, there has been little development of the RF SQUID in the last decade.

In this paper an overview is given of the current state of the SQUID art. We cannot hope to present all of the SQUIDs and SQUID-based instruments that have been made or, even less, all of the applications in which they have been used successfully. In Section II we describe how dc SQUIDs are made and operated, and the limitations imposed by noise. Section III contains a similar, brief description of the properties of RF SQUIDs. In Section IV, a selection of instruments based on SQUIDs is described together with some of their applications. Section $V$ is a discussion of the impact of high-temperature superconductivity on SQUIDs and of future prospects in this area. Section VI contains a few concluding remarks.

\section{THE DC SQUID}

\section{A. Theory of Operation}

The essence of the dc SQUID [3] is shown in Fig. 1(a). Two Josephson junctions are connected in parallel on a superconducting loop of inductance $L$. Each junction is resistively shunted [6], [7] to eliminate hysteresis on the current- 


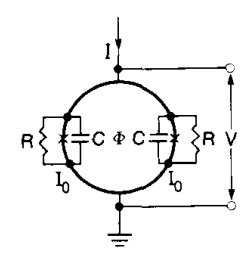

(a)

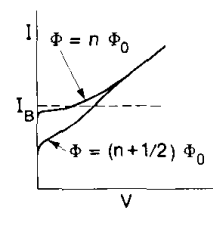

(b)

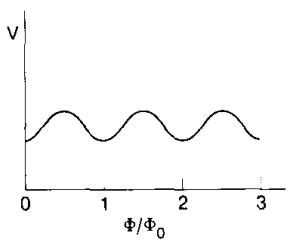

(c)
Fig. 1. (a) DC SQUID. (b) I-V characteristics. (c) $V$ versus $\Phi /$ $\Phi_{0}$ at constant bias current $I_{B}$.

voltage $(I-V)$ characteristics, which are shown in Fig. 1(b) for $\Phi=n \Phi_{0}$ and $\left(n+\frac{1}{2}\right) \Phi_{0}$, where $\Phi$ is the external flux applied to the loop and $n$ is an integer. If we bias the SQUID with a constant current, the voltage across the SQUID oscillates as we increase $\Phi$ steadily, as indicated in Fig. 1 (c). The period is $\Phi_{0}$. The SQUID is generally operated on the steep part of the $V-\Phi$ curve where the transfer coefficient $V_{\Phi} \equiv\left|(\partial V / \partial \Phi)_{l}\right|$ is a maximum. Thus, the SQUID produces an output voltage in response to a small input flux $\delta \Phi\left(\ll \Phi_{0}\right)$ and is effectively a flux-to-voltage transducer. The resolution can be characterized by the equivalent flux noise $\Phi_{N}(t)$, which has a spectral density

$$
S_{\Phi}(f)=\frac{S_{V}(f)}{V_{\Phi}^{2}}
$$

at frequency $f$. Here $S_{V}(f)$ is the spectral density of the voltage noise across the SQUID at fixed current bias. A more useful characterization, however, is in terms of the flux noise energy associated with $S_{\Phi}(f)$,

$$
\epsilon(f)=\frac{S_{\Phi}(f)}{2 L}
$$

where $L$ is the inductance of the loop.

Thermal noise imposes two constraints on the parameters of the SQUID. First, the coupling energy $I_{0} \Phi_{0} / 2 \pi$ of each junction must be significantly greater than $k_{B} T$. A computer analysis yields [8]

$$
\frac{l_{0} \Phi_{0}}{2 \pi} \geq 5 k_{B} T
$$

At $4.2 \mathrm{~K}$, Eq. (3) implies $I_{0} \geq 0.9 \mu \mathrm{A}$. Second, the root mean square thermal noise flux in the loop, $\left\langle\Phi_{N}^{2}\right\rangle^{1 / 2}=\left(k_{B} T L\right)^{1 / 2}$, must be significantly less than $\Phi_{0}$. The same computer analysis [8] leads to

$$
L \leq \frac{\Phi_{0}^{2}}{5 k_{B} T} .
$$

This constraint implies $L \leqslant 15 \mathrm{nH}$ at $4.2 \mathrm{~K}$.

We now outline the signal and noise properties of the SQUID. Each junction in Fig. 1(a) has a critical current $I_{0}$, a self-capacitance $C$, and a resistive shunt $R$ chosen so that $\beta_{C} \equiv 2 \pi I_{0} R^{2} C / \Phi_{0} \leqslant 1$. The two resistors generate statistically independent Nyquist noise currents $I_{N 1}(t)$ and $I_{N 2}(t)$, each with a spectral density $4 k_{B} T R$ at temperature $T$. The differences in the phase across each junction, $\delta_{1}(t)$ and $\delta_{2}(t)$, obey the following equations [9]-[11]:

$$
\begin{aligned}
& V=\frac{\hbar}{4 \mathrm{e}}\left(\dot{\delta}_{1}+\dot{\delta}_{2}\right) \\
& J=\frac{\Phi_{0}}{2 \pi L}\left(\delta_{1}-\delta_{2}-\frac{2 \pi \Phi}{\Phi_{0}}\right)
\end{aligned}
$$

$$
\begin{aligned}
& \frac{\hbar C}{2 e} \ddot{\delta}_{1}+\frac{\hbar}{2 e R} \dot{\delta}_{1}=\frac{1}{2}-J-I_{0} \sin \delta_{1}+I_{N 1} \\
& \frac{\hbar C}{2 e} \ddot{\delta}_{2}+\frac{\hbar}{2 e R} \dot{\delta}_{2}=\frac{1}{2}+J-I_{0} \sin \delta_{2}+I_{N_{2}} .
\end{aligned}
$$

Equation (5) relates the voltage to the average rate of change of phase, Eq. (6) expresses fluxoid quantization, and Eqs. (7) and (8) are Langevin equations coupled via J. These equations have been solved numerically for a limited range of values of the noise parameter $\Gamma=2 \pi k_{B} T / I_{0} \Phi_{0}$, reduced inductance $\beta=2 L I_{0} / \Phi_{0}$, and hysteresis parameter $\beta_{C}$. For typical SQUIDs in the ${ }^{4} \mathrm{He}$ temperature range, $\Gamma=0.05$. One computes the time averaged voltage $V$ versus $\Phi$, and hence finds $V_{\Phi}$, which, for a given value of $\Phi$, peaks smoothly as a function of bias current. The transfer function exhibits a shallow maximum around $(2 n+1) \Phi_{0} / 4$. One computes the noise voltage for a given value of $\Phi$ as a function of $I$ and finds that the spectral density is white at frequencies much less than the Josephson frequency. For each value of $\Phi$, the noise voltage peaks smoothly at the value of $I$ where $V_{\Phi}$ is a maximum. From these simulations, one finds that the noise energy has a minimum when $\beta \simeq 1$. For $\beta=1, \Gamma=$ $0.05, \Phi=(2 n+1) \Phi_{0} / 4$, and for the value of $I$ at which $V_{\Phi}$ is a maximum, the results can be summarized as follows:

$$
\begin{aligned}
V_{\Phi} & \simeq \frac{R}{L} \\
S_{V}(f) & \simeq 16 k_{B} T R \\
\epsilon(f) & \simeq \frac{9 k_{B} T L}{R} .
\end{aligned}
$$

It is often convenient to eliminate $R$ from Eq. (11) using the expression $R=\left(\beta_{C} \Phi_{0} / 2 \pi I_{0} C\right)^{1 / 2}$. We find

$$
\epsilon(f) \simeq 16 k_{B} T\left(\frac{L C}{\beta_{C}}\right)^{1 / 2}, \quad \beta_{C} \leqslant 1 .
$$

Equation (12) gives a clear prescription for improving the resolution: one should reduce $T, L$, and $C$. A large number of SQUIDs with a wide range of parameters have been tested and found to have noise energies generally in good agreement with the predicted values. It is common practice to quote the noise energy of SQUIDs in units of $h\left(\approx 10^{-34}\right.$, $\cdot \mathrm{s}=10^{-34} \mathrm{~J} \cdot \mathrm{Hz}^{-1}$ )

\section{B. Practical dc SQUIDs}

Modern dc SQUIDs are invariably made from thin films, with the aid of either photolithography or electron beam lithography. A major concern in the design is the need to couple an input coil inductively to the SQUID with rather high efficiency. This problem was elegantly solved by Ketchen and Jaycox [12], [13], who introduced the idea of depositing a spiral input coil on a SQUID in a square washer configuration. The coil is separated from the SQUID with an insulating layer. The version [14] of this design made at the University of California in Berkeley is shown in Fig. 2. These devices are made in batches of 36 on 50-mm-diameter oxidized silicon wafers in the following way. First, a 30-nmthick Au ( $25 \mathrm{wt}-\% \mathrm{Cu}$ ) film is deposited and patterned to form the resistive shunts. Next, we sputter a 100 -nm-thick $\mathrm{Nb}$ film and etch it to form the SQUID loop and a strip that eventually contacts the inner end of the spiral coil. The third 


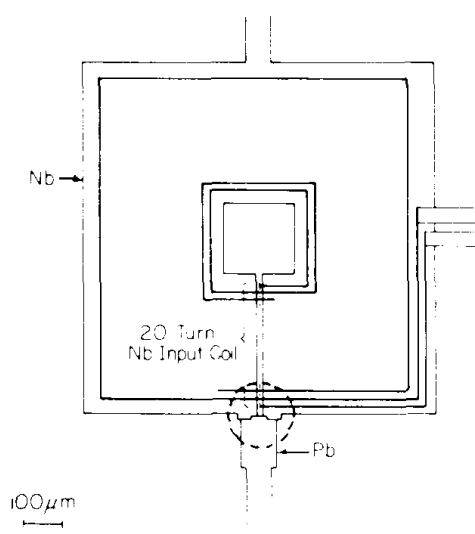

(a)

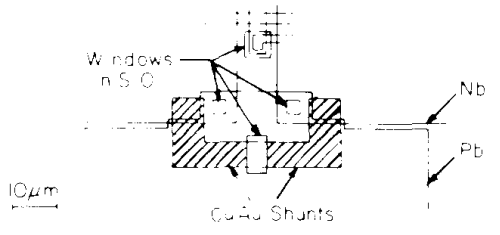

(b)

Fig. 2. (a) Configuration of planar de SQUHI) with overlaid spiral input coil. (b) Expanded view of junctions and shunts.

film is a $200-\mathrm{nm} \mathrm{SiO}$ layer with 2- $\mu$ m-diameter windows for the junctions, a larger window to give access to the $\mathrm{CuAu}$ shunt, and a window at each end of the Nb strip to provide connections to the spiral coil. The next step is to deposit and lift off the 300-nm-thick Nb spiral coil, which has 4, 20, or 50 turns. At this point, we usually dice the water into chips, each with a single SQUID, which is completed individually. The device is ion milled to clean the exposed areas of $\mathrm{Nb}$ and $\mathrm{CuAu}$. We have two procedures for forming the oxide barrier. In one, we oxidize the $\mathrm{Nb}$ in an RF discharge in Ar containing 5 vol- \% $\mathrm{O}_{2}$ and deposit the $300-\mathrm{nm} \mathrm{Pb}(5$ wt- $\%$ In) counterelectrode, which completes the junctions and makes contact with the shunts. In the other process, we deposit approximately $6 \mathrm{~nm}$ of $\mathrm{Al}$ and form $\mathrm{Al}_{2} \mathrm{O}$, by exposing [15] it to $\mathrm{O}_{2}$. The completed SQUID and a scanning electron micrograph of the junctions are shown in Fig. 3. The shunt resistance $R$ is typically $8 \Omega$, the capacitance $C$ about $0.5 \mathrm{pF}$, and the inductance $L$ about $0.4 \mathrm{nH}$. For a 50 turn input coil, one finds $L_{1}=800 \mathrm{nH}$ and $M_{1}=16 \mathrm{nH}$.

References [16]-[21] are a selection of papers describing SQUIDs fabricated on the Ketchen-Jaycox design. Some of the devices involve edge junctions, in which the counterelectrode is a strip making a tunneling contact to the base electrode only at the edge. This technique enables one to make junctions with a small area and thus a small self-capacitance without resorting to electron-beam lithography. However, stray capacitances are often critically important. As has been emphasized by a number of authors, parasitic capacitance between the square washer and the input coil can produce resonances, which in turn induce structure on the $I-V$ characteristics and give rise to excess noise. One way to reduce these effects is to lower the shunt resistance to increase the damping. An alternative approach is to couple the SQUID to the signal via an intermediary superconducting transformer [20], so that the number of lums on the SQUID washer and the parasitic capacitance are reduced. Knuutila et al. [21] successiully damped the resonances in the input coil by terminating the stripline with a matched resistor. An alternative coupling scheme has been adopted by Carelliand Foglietti [22], who fabricated thin-tilm SQ QUID; with many loops in parallel. The loops are coupled to a thinfilm input coil surrounding them.

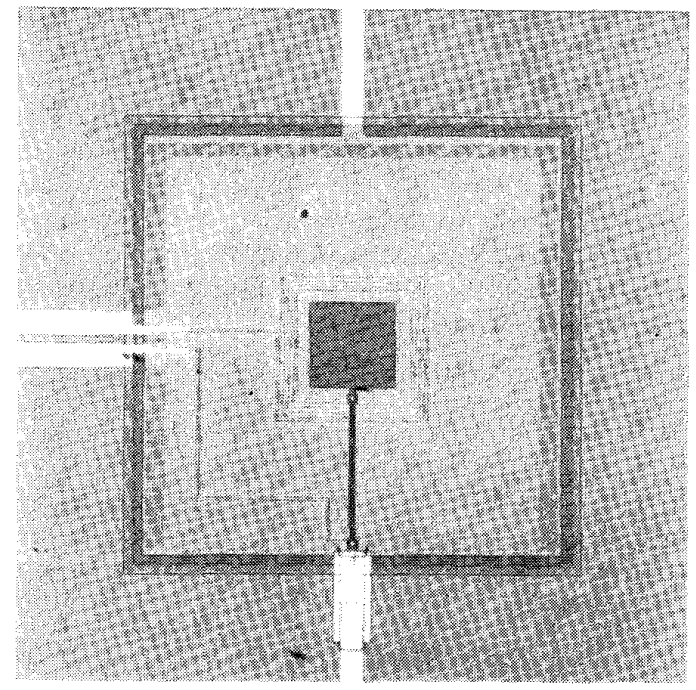

(a)

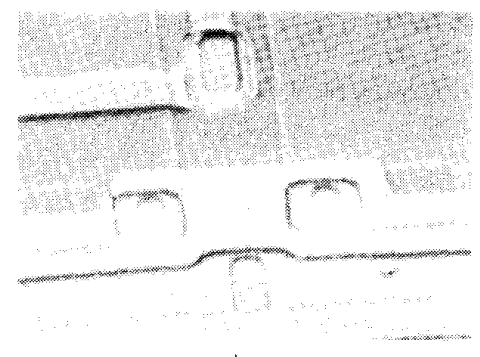

(b)

Fig. 3. (a) Planar de SQUID made at Univereity of California at Berkeles, with 4-turn input coil: square washer is approximately $1 \mathrm{~mm}$ acrous, (b) Electron nicrograph of junctions prior to deposition of counterelestrode; each junction is approximately 3 m ac ross

\section{Flux-Locked Loop}

In most, although not all, practical applications one uses the SQUID in a feedback circuit as a null detector of magnetic flux [23]. One applies a modulating flux to the SQUID 
with a peak-to-peak amplitude $\Phi_{0} / 2$ and a frequency $f_{m}$ usually between 100 and $500 \mathrm{kHz}$, as indicated in Fig. 4. If the quasistatic flux in the SQUID is exactly $n \Phi_{0}$, the resulting voltage is a rectified version of the input signal, that is, it contains only the frequency $2 f_{m}$ [Fig. 4(a)]. If this voltage is

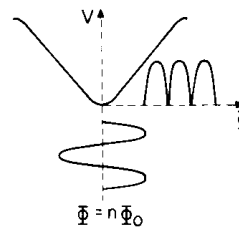

(a)

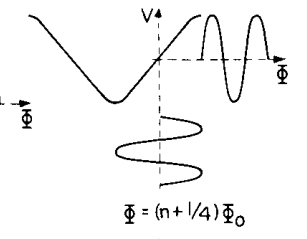

(b)

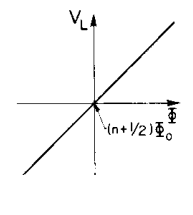

(c)
Fig. 4. Flux modulation scheme showing voltage across SQUID for (a) $\Phi=n \Phi_{0}$ and (b) $\Phi=\left(n+\frac{1}{4}\right) \Phi_{0}$. (c) Output $V_{t}$ from lock-in detector versus $\Phi$.

sent through a lock-in detector referenced to the fundamental frequency $f_{m}$, the output will be zero. On the other hand, if the quasistatic flux is $\left(n+\frac{1}{4}\right) \Phi_{0}$, the voltage across the SQUID is at frequency $f_{m}$ [Fig. 4(b)], and the output from the lock-in will be a maximum. Thus, as one increases the flux from $n \Phi_{0}$ to $\left(n+\frac{1}{4}\right) \Phi_{0}$, the output from the lock-in will increase steadily; if one reduces the flux from $n \Phi_{0}$ to $\left(n-\frac{1}{4}\right) \Phi_{0}$, the output will increase in the negative direction [Fig. 4(c)].

The alternating voltage across the SQUID is coupled to a low-noise preamplifier, usually at room temperature, via either a cooled transformer [24] or a cooled $L C$ series-resonant circuit [23]. The first presents an impedance $N^{2} R_{d}$ to the preamplifier and the second an impedance $Q^{2} R_{d}$, where $R_{d}$ is the dynamic resistance of the SQUID at the bias point, $N$ is the turns ratio of the transformer, and $Q$ is the quality factor of the tank circuit. The value of $N$ or $Q$ is chosen to optimize the noise temperature of the preamplifier; with careful design, the noise from the amplifier can be appreciably less than that from the SQUID at $4.2 \mathrm{~K}$.

Fig. 5 shows a typical flux-locked loop in which the SQUID is coupled to the preamplifier via a cooled transformer. An

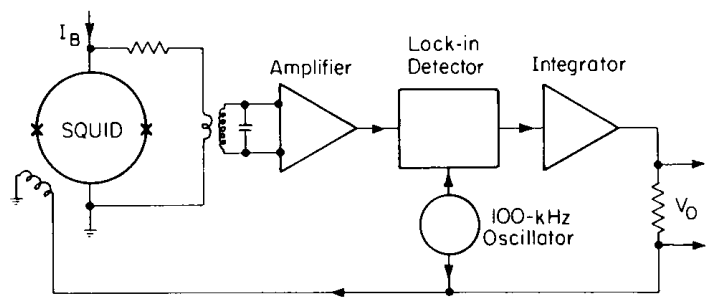

Fig. 5. Modulation and feedback circuit for dc SQUID.

oscillator applies a modulating flux to the SQUID. After amplification, the signal from the SQUID is lock-in detected and sent through an integrating circuit. The smoothed output is connected to the modulation and feedback coil via a large series resistor $R_{f}$. Thus, if one applies a flux $\delta \Phi$ to the SQUID, the feedback circuit will generate an opposing flux $-\delta \Phi$, and a voltage proportional to $\delta \Phi$ appears across $R_{f}$ This technique enables one to measure changes in flux ranging from much less than a single flux quantum to many flux quanta. The use of a modulating flux eliminates $1 / f$ noise and drift in the bias current and preamplifier. Using a modulation frequency of $500 \mathrm{kHz}$, a double transformer between the SQUID and the preamplifier, and a two-pole integrator, Wellstood et al. [14] achieved a dynamic range of $\pm 2 \times 10^{7}$ $\mathrm{Hz}^{1 / 2}$ for signal frequencies up to $6 \mathrm{kHz}$, a frequency response from 0 to $70 \mathrm{kHz}( \pm 3 \mathrm{~dB})$, and a maximum slew rate of $3 \times 10^{6} \Phi_{0} \mathrm{~s}^{-1}$.

\section{Thermal Noise in the dC SQUID: Experiment}

One determines the spectral density of the equivalent flux noise in the SQUID by connecting a spectrum analyzer to the output of the flux-locked loop. A representative power spectrum [25] is shown in Fig. 6. Above a $1 / f$ noise

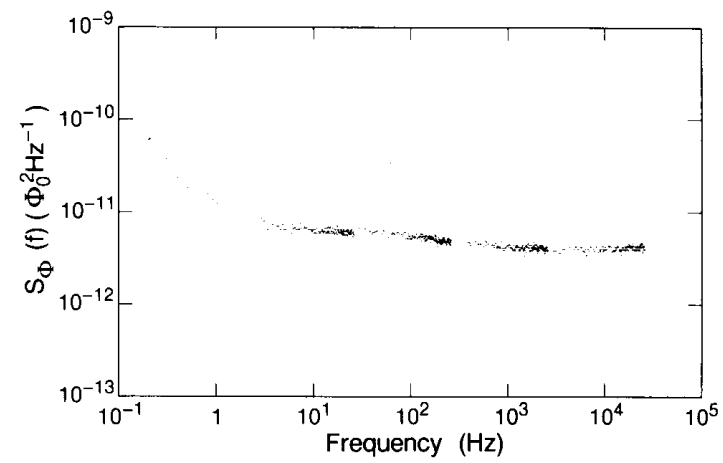

Fig. 6. Spectral density of equivalent flux noise for $\mathrm{dc}$ SQUID with $\mathrm{Pb}$ body. $L=0.2 \mathrm{nH} ; R=5 \Omega ; T=4.2 \mathrm{~K}$. (Courtesy of F. C. Wellstood.)

region, the noise is white at frequencies up to the roll-off of the feedback circuit. In this particular example, with $L$ $=200 \mathrm{pH}$ and $R=5 \Omega$, the measured white flux noise was $S_{\Phi}^{1 / 2}=(1.9 \pm 0.1) \times 10^{-6} \Phi_{0} \mathrm{~Hz}^{-1 / 2}$, in reasonable agreement with the predictions of Eqs. (9) and (10), $1.3 \times 10^{-6} \Phi_{0}$ $\mathrm{Hz}^{-1 / 2}$. The corresponding flux noise energy was $4 \times 10^{-32}$ $\mathrm{J} \cdot \mathrm{Hz} \approx 400 \hbar$. Many groups have achieved noise energies that are comparable or, with lower values of $L$ or $C$, somewhat better.

Rather recently, Wellstood et al. [26] have operated SQUIDs in a dilution refrigerator at temperatures $T$ below $1 \mathrm{~K}$, using a second dc SQUID as a preamplifier. They found that the noise energy scaled rather accurately with $T$ at temperatures down to about $150 \mathrm{mK}$, below which the noise energy became nearly constant. This saturation was traced to heating in the resistive shunts, which prevented them from cooling much below $150 \mathrm{mK}$. This heating is actually a hot electron effect [27], [28]. The bottleneck in the cooling process is the rate at which the electrons can transfer energy to the phonons which, in turn, transfer energy to the substrate. The temperature of the shunts was lowered by connecting each of them to a CuAu "cooling fin" of large volume. The hot electrons diffuse into the fins, where they rapidly transfer energy to other electrons. Since the "reaction volume ${ }^{\prime \prime}$ is now greatly increased, the numbers of electrons and phonons interacting are also increased, and the electron gas is cooled more effectively. In this way, the effective electron temperature was reduced to about $50 \mathrm{mK}$ when the SQUID was at a bath temperature of $20 \mathrm{mK}$, with a concommitant reduction in $\epsilon$ to about $5 \hbar$. Very recently 
Ketchen et al. [29] have achieved a noise energy of about $3 h$ at $0.3 \mathrm{~K}$ in a SQUID with $L=100 \mathrm{pH}$ and $C=0.14 \mathrm{pF}$.

\section{E. $7 / \mathrm{f}$ Noise in dc SQUIDs}

The white noise in dc SQUIDs is well understood. However, some applications of SQUIDs, such as neuromagnetism, require good resolution at frequencies down to $0.1 \mathrm{~Hz}$ or less, and the level of the $1 / f$, or "flicker," noise becomes very important.

There are at least two separate sources of $1 / f$ noise in the dc SQUID [30]. The first arises from $1 / f$ noise in the critical current of the Josephson junctions, and the mechanism for this process is reasonably well understood [31]. In the process of tunneling through the barrier, an electron becomes trapped for a while and is subsequently released. While the trap is occupied, there is a local change in the height of the tunnel barrier and hence in the critical current density of that region. As a result, the presence of a single trap causes the critical current of the junction to switch randomly back and forth between two values, producing a random tele graph signal. If the mean time between pulses is $\tau$, the spectral density of this process is a Lorentzian,

$$
S(f) \propto \frac{\tau}{1+(2 \pi f \tau)^{2}}
$$

namely, white at low frequencies and falling off as $1 / f^{2}$ at frequencies above $1 / 2 \pi \tau$. In many cases, the trapping process is thermally activated, and $\tau$ is of the form

$$
\tau=\tau_{0} \exp \left(\frac{E}{k_{B} T}\right)
$$

where $\tau_{0}$ is a constant and $E$ is the barrier height.

In general, there may be several traps in the junction, each with its own characteristic time $\tau_{i}$. One can superimpose the trapping processes, assuming them to be statistically independent, to obtain a spectral density [32]

$$
S(f) \propto \int d E D(E)\left|\frac{\tau_{0} \exp \left(E / k_{B} T\right)}{1+\left(2 \pi f \tau_{0}\right)^{2} \exp \left(2 E / k_{B} T\right)}\right|
$$

where $D(E)$ is the distribution of activation energies. The term in square brackets is a strongly peaked function of $E$ centered at $\tilde{E} \equiv k_{B} T \ln \left(1 / 2 \pi f \tau_{0}\right)$ and with a width $\sim k_{B} T$. Thus, at a given temperature, only traps with energies within a range $k_{B} T$ of $\tilde{E}$ contribute significantly to the noise. If one now assumes $D(E)$ is broad with respect to $k_{B} T$, one can take $D(\tilde{E})$ outside the integral and perform the integration to obtain

$$
S(f, T) \propto \frac{k_{B} T}{f} D(\tilde{E}) .
$$

In fact, one obtains a $1 / f$-like spectrum from just a few traps.

The magnitude of the $1 / f$ noise in the critical current depends strongly on the quality of the junction as measured by the current leakage at voltages below $\left(\Delta_{1}+\Delta_{2}\right)$ f $e$, where $\Delta_{1}$ and $\Delta_{2}$ are the energy gaps of the two superconductors. Traps in the barrier enable electrons to tunnel in this voltage range, a process producing both leakage current and $1 / f$ noise. Thus, for a given technology, junctions with low subgap leakage currents will have low $1 / f$ noise.

The second source of $1 / f$ noise in SQUIDs appears to arise from the motion of flux lines trapped in the body of the SQUID [30] and is less well understood than the critical cur- rent noise. This mechanism manifests itself as a flux noise. For all practical purposes the noise source behaves as if an external flux noise were applied to the SQUID. Thus, the spectral density of the $1 / f$ flux noise scales as $V_{\Phi}^{2}$ and, in particular, vanishes at $\Phi=\left(n \pm \frac{1}{2}\right) \Phi_{0}$ where $V_{\Phi}=0$. By contrast, critical current noise is still present when $V_{\Phi}=0$, although its magnitude does depend on the applied flux. The level of $1 / f$ flux noise appears to depend strongly on the microstructure of the thin films. For example, SQUIDs fabricated at Berkeley with $\mathrm{Nb}$ loops sputtered under a particular set of conditions show $1 / f$ flux noise levels of typically $10^{-10} \Phi_{0}^{2} \mathrm{~Hz}^{-1}$ at $1 \mathrm{~Hz}$ [30]. On the other hand, SQUIDs with $\mathrm{Pb}$ loops in exactly the same geometry exhibit a $1 / f$ noise level of about $2 \times 10^{-12} \Phi_{0}^{2} \mathrm{~Hz}^{-1}$ at $1 \mathrm{~Hz}$, arising from critical current fluctuations. Tesche et al. [33] reported a $1 / f$ noise level in Nb-based SQUIDs of about $3 \times 10^{-13} \Phi_{0}^{2} \mathrm{~Hz}^{-1}$, while Foglietti et al. [34] found a critical current $1 / f$ noise corresponding to $2 \times 10^{-12} \Phi_{0}^{2} \mathrm{~Hz}^{-1}$, also in Nb-based devices. Thus, we conclude that the quality of the $\mathrm{Nb}$ films plays a significant role in the level of $1 /$ flux noise. It is of considerable fundamental and practical interest to understand the mechanism in detail.

There is an important practical difference between the two sources of $1 / f$ noise; critical current noise can be reduced by a suitable modulation scheme whereas flux noise cannot [30], [34], [35]. These schemes involve switching the bias current as well as the flux, and at least two methods have been used successfully. The resulting reduction in the spectral density of the $1 /$ f noise due to critical current fluctuations may be an order of magnitude or more.

\section{F. Alternative Read-Out Schemes}

Although the flux modulation method described in Section II-C has been used successfully for many years, alternative schemes have recently been developed. Fujimaki and coworkers [36] and Drung and coworkers [37] have devised schemes in which the output from the SQUID is sensed digitally and fed back as an analog signal to the SQUID to flux-lock the loop. Fujimaki et al. [36] used Josephson digital circuitry to integrate their feedback system on the same chip as the SQUID so that the flux-locked signal was available directly from the cryostat. The system of Drung and coworkers, however, is currently the more sensitive with a flux resolution of about $10^{-6} \Phi_{0} \mathrm{~Hz}^{-1 / 2}$ in a 50-pH SQUID. These workers were also able to reduce the $1 / f$ noise in the system using a modified version of the modulation scheme of Foglietti et al. [34]. Although they need further development, cryogenic digital feedback schemes offer several advantages: they are compact, produce a digital output for transmission to room temperature, offer wide flux-locked bandwidths, and need not add any noise to the intrinsic noise of the SQUID.

In yet another system, Mück and Heiden [38] have operated a dc SQUID with hysteretic junctions in a relaxation oscillator. The oscillation frequency depends on the flux in the SQUID, reaching a maximum at $\left(n+\frac{1}{2}\right) \Phi_{0}$ and a minimum at $n \Phi_{0}$. A typical frequency modulation is $100 \mathrm{kHz}$ at an operating frequency of $10 \mathrm{MHz}$. This technique produces large voltages across the SQUID so that no matching network to the room temperature electronics is required. The room temperature electronics are simple and compact, and the resolution is about $10^{-5} \Phi_{0} \mathrm{~Hz}^{-1 / 2}$ for a SQUID at 4.2 $\mathrm{K}$ with an inductance estimated to be about $80 \mathrm{pH}$. 


\section{THE RF SQUID}

\section{A. Principles of Operation}

Although the RF SQUID is still the more widely used device because of its long-standing commercial availability, it has seen very little development in recent years. For this reason a rather brief account of its principles and noise limitations is given here and the reader is referred to more detailed descriptions in earlier reviews [39], [40].

The RF SQUID [4], [5] shown in Fig. 7 consists of a superconducting loop of inductance $L$, interrupted by a single

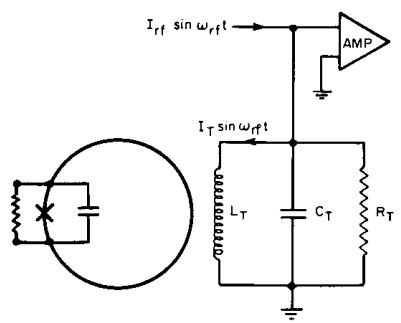

Fig. 7. RF SQUID inductively coupled to resonant tank circuit.

Josephson junction with critical current $I_{0}$ and a nonhysteretic current-voltage characteristic. The critical current is chosen so that $L I_{0} \approx \Phi_{0}$. The loop is inductively coupled with a coupling coefficient $K$ to the inductor of a tank circuit that is excited by an RF current at its resonant frequency of typically 20 or $30 \mathrm{MHz}$. Optimum performance is obtained when $K^{2} Q \approx 1$. To observe the operation of the SQUID, one monitors the amplitudes of the RF current $I_{\mathrm{RF}}$ and voltage $V_{R F}$ obtained by demodulating the RF signals. A typical result is shown in Fig. 8. For $\Phi=n \Phi_{0}$ one observes a series of steps

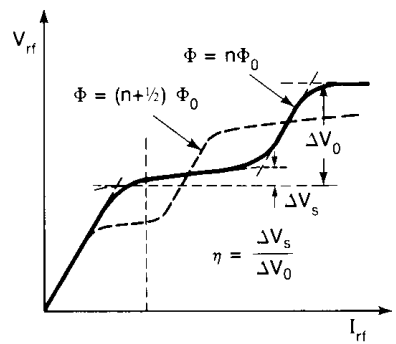

Fig. 8. Demodulated voltage $V_{\mathrm{RF}}$ versus demodulated current $l_{\mathrm{BF}}$ for $\Phi=n \Phi_{0},\left(n+\frac{1}{2}\right) \Phi_{0}$. Vertical dashed line indicates amplitude of RF bias current for normal operation. Slope parameter $\eta$ is defined as shown.

and risers; as one increases (or decreases) the flux, each step splits into two, with a maximum separation in voltage at $\left(n+\frac{1}{2}\right) \Phi_{0}$. As the flux is further increased to $(n+1) \Phi_{0}$ or decreased to $(n-1) \Phi_{0}$, the $V_{\mathrm{RF}}-I_{\mathrm{RF}}$ curve is restored to the solid line in Fig. 8. Thus, at constant RF current bias (vertical dashed line in Fig. 8), the demodulated voltage is periodic in $\Phi$, with an optimum transfer function

$$
V_{\phi} \approx \omega_{\mathrm{RF}}\left(\frac{Q L_{T}}{L}\right)^{1 / 2}
$$

where $L_{T}$ is the tank circuit inductance. A modulating flux, typically at $100 \mathrm{kHz}$ and with a peak-to-peak amplitude of $\Phi_{0} / 2$, is also applied to the SQUID, just as in the case of the dc SQUID. The voltage produced by this modulation is lockin detected, integrated, and fed back as a current into the modulation coil to flux-lock the SQUID.

\section{B. Theory of Noise in the RF SQUID}

A detailed theory has been developed for noise in the RF SQUID [41]-[49]. In contrast to the case for the dc SQUID, noise contributions from the tank circuit and preamplifier are also important. Thermal fluctuations have two effects on the intrinsic noise of the SQUID when it is driven with an RF flux. First, noise is induced on the RF voltage across the tank circuit, giving an equivalent intrinsic flux noise spectral density [42], [46]

$$
S_{\Phi}^{(i)} \approx \frac{\left(L I_{0}\right)^{2}}{\omega_{\mathrm{RF}}}\left(\frac{2 \pi k_{B} T}{I_{0} \Phi_{0}}\right)^{4 / 3} .
$$

Second, the noise causes the steps to tilt. Jackel and Buhrman [43] introduced the slope parameter $\eta$ defined in Fig. 8 and showed that it was related to $S_{\phi}^{(i)}$ by the relation

$$
\eta^{2} \approx \frac{S_{\Phi}^{(i)} \omega_{\mathrm{RF}}}{\pi \Phi_{0}^{2}}
$$

provided $\eta$ was not too large. This relation is well verified experimentally.

The noise temperature $T_{a}$ of typical RF amplifiers operated at room temperature is substantially higher than that of amplifiers operated at a few hundred kilohertz, and is therefore not negligible for RF SQUIDs operated at liquid ${ }^{4}$ He temperatures. Furthermore, part of the coaxial line connecting the tank circuit to the preamplifier is at room temperature. Since the capacitances of the line and the amplifier are a substantial fraction of the capacitance of the tank circuit, part of the resistance damping the tank circuit is well above the bath temperature. As a result there is an additional contribution to the noise, which we combine with the preamplifier noise to produce an effective noise temperature $T_{a}^{\text {eff }}$. The noise energy contributed by these extrinsic sources can be shown to be $2 \pi \eta k_{B} T_{d}^{\text {eff }} / \omega_{\mathrm{RF}}$ [43], [47]. Combining this contribution with the intrinsic noise, one finds

$$
\epsilon \approx \frac{1}{\omega_{\mathrm{RF}}}\left(\frac{\pi \eta^{2} \Phi_{0}^{2}}{2 L}+2 \pi \eta k_{B} T_{a}^{\mathrm{eff}}\right) .
$$

Equation (20) shows that $\epsilon$ scales as $1 / \omega_{\mathrm{RF}}$, but one should bear in mind that $T_{\mathrm{a}}$ tends to increase with $\omega_{\mathrm{RF}}$. Nonetheless, improvements in performance have been achieved by operating the SQUID at much higher frequencies [50], [51] than the usual 20 or $30 \mathrm{MHz}$. One can also reduce $T_{a}^{\text {eff }}$ by cooling the preamplifier [50], [53], thereby reducing $T_{a}$ and reducing the temperature of the tank circuit to that of the bath.

\section{Practical RF SQUIDs}

Although less sensitive than the dC SQUID, the RF SQUID is entirely adequate for a wide range of applications. It is more widely used than the dC SQUID for the simple reason that reliable, easy-to-operate devices have been commer-

${ }^{1}$ For a review, see [52]. 
cially available since the early 1970 s, notably from BTi (formerly SHE). Fig. 9 shows a cutaway drawing of this device [35], which has a toroidal configuration machined from $\mathrm{Nb}$. One way to understand this geometry is to imagine rotating

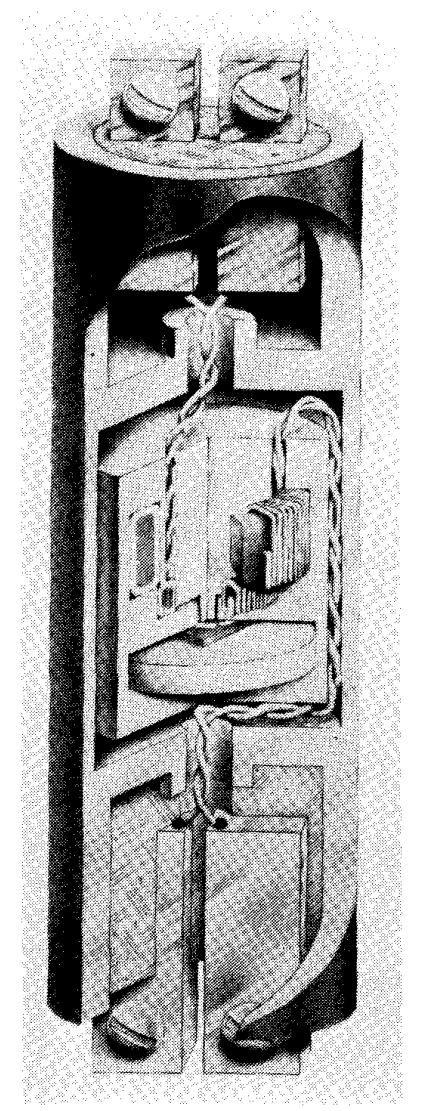

Fig. 9. Cutaway drawing of toroidal SQUID. (Courtesy of BTi, Inc.)

the SQUID in Fig. 7 through $360^{\circ}$ about a line running through the junction from top to bottom. This procedure produces a toroidal cavity concerned at its center by the junction. If one places a toroidal coil in this cavity, a current in the coil produces a flux that is tightly coupled to the SQUID. In Fig. 9 there are actually two such cavities, one containing the tank circuit-modulation-feedback coil and the other the input coil. This separation eliminates crosstalk between the two coils. Leads to the two coils are brought out via screw terminals. The junction is made from thin films of $\mathrm{Nb}$. This device is self-shielding against external magnetic field fluctuations and has proven to be reliable and convenient to use. In particular, the $\mathrm{Nb}$ input terminals enable one to connect different input circuits in a straightforward way. A typical device has a white noise energy of $5 \times 10^{-29} \mathrm{~J} \cdot \mathrm{Hz}^{-1}$, with a $1 / \mathrm{f}$ noise energy of perhaps $10^{-28}$ J. $\mathrm{Hz}^{-1}$ at $0.1 \mathrm{~Hz}$.

\section{SQUID-BASED INSTRUMENTS}

Both dc and RF SQUIDs are used as sensors in a far-ranging assortment of instruments, some of which are discussed briefly in this section. Our selection is far from exhaustive, but does include the more commonly used instruments.

Each instrument involves a circuit attached to the input coil of the SQUID. We should recognize from the outset that, in general, the presence of the input circuit influences both the signal and the noise properties of the SQUID, while the SQUID, in turn, reflects a complex impedance into the input. Because the SQUID is a nonlinear device, a full description of the interactions is complicated, and we shall not go into the details here. However, one aspect of this interaction, first pointed out by Zimmerman [54], is easy to understand. Suppose we connect a superconducting pickup loop of inductance $L_{p}$ to the input coil of inductance $L_{i}$, to form a magnetometer, as shown in Fig. 10(a). It is easy

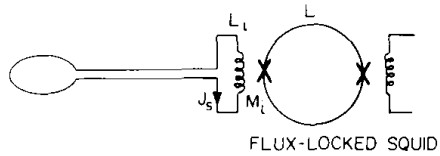

(a)

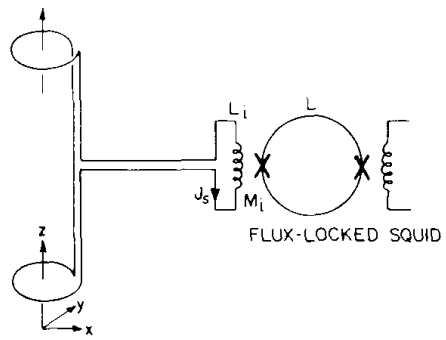

(b)

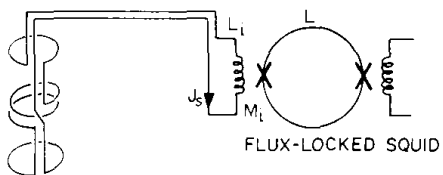

(c)

Fig. 10. Superconducting flux transformers. (a) Magnetometer. (b) First-derivative gradiometer. (c) Second-derivative gradiometer.

to show that the SQUID inductance $L$ is reduced to the value

$$
L^{\prime}=L\left(1-\frac{\alpha^{2} L_{i}}{L_{i}+L_{p}}\right)
$$

where $\alpha^{2}$ is the coupling coefficient between $L$ and $L_{i}$. We have neglected any stray inductance in the leads connecting $L_{i}$ and $L_{p}$, and any stray capacitance. The reduction in $L$ tends to increase the transfer coefficient of both the dc SQUID [Eq. (9)] and the RF SQUID [Eq. (17)]. The reduction in $L$ and the change in noise properties may affect the results of optimization schemes appreciably under certain circumstances [25], but we shall not discuss these issues here.

\section{A. Magnetometers and Gradiometers}

One of the simplest instruments is the magnetometer [Fig. 10(a)]. A pickup loop is connected across the input coil to make a superconducting flux transformer. The SQUID and 
the input coil are generally enclosed in a superconducting shield. If one applies a magnetic flux $\delta \Phi^{(p)}$ to the pickup loop, flux quantization requires that

$$
\delta \Phi^{(p)}+\left(L_{i}+L_{p}\right) J_{S}=0
$$

where $J_{S}$ is the supercurrent induced in the transformer. We have neglected the effects of the SQUID on the input circuit. The flux coupled into the SQUID, which we assume to be in a flux-locked loop, is $\delta \Phi=M_{i}\left|J_{S}\right|=M_{i} \delta \Phi^{(p)} /\left(L_{i}+\right.$ $\left.L_{p}\right)$. We find the minimum detectable value of $\delta \Phi^{(p)}$ by equating $\delta \Phi$ with the equivalent flux noise of the SQUID. Defining $S_{\Phi}^{(\rho)}$ as the spectral density of the equivalent flux noise referred to the pickup loop, we find

$$
S_{\Phi}^{(p)}=\frac{\left(L_{p}+L_{i}\right)^{2}}{M_{i}^{2}} S_{\Phi}
$$

Introducing the equivalent noise energy referred to the pickup loop, we obtain

$$
\frac{S_{\Phi}^{(p)}}{2 L_{p}}=\frac{\left(L_{p}+L_{i}\right)^{2}}{L_{i} L_{p}} \frac{S_{\Phi}}{2 \alpha^{2} L} .
$$

We observe that Eq. (24) has the minimum value

$$
\frac{S_{\Phi}^{(p)}}{2 L_{p}}=\frac{4 \epsilon(f)}{\alpha^{2}}
$$

when $L_{i}=L_{p}$. Thus, a fraction $\alpha^{2} / 4$ of the energy in the pickup loop is transferred to the SQUID. In this derivation we have neglected noise currents in the input circuit arising from noise in the SQUID, the fact that the input circuit reduces the SQUID inductance, and any possible coupling between the feedback coil of the SQUID and the input circuit.

Having obtained the flux resolution for $L_{i}=L_{p}$, we can immediately write down the corresponding magnetic field resolution $B_{N}^{(p)}=\left(S_{\Phi}^{(p)}\right)^{1 / 2} / \pi r_{p}^{2}$, where $r_{p}$ is the radius of the pickup loop,

$$
B_{N}^{(p)}=\frac{2 \sqrt{2} L_{p}^{1 / 2} \epsilon^{1 / 2}}{\pi r_{p}^{2} \alpha} .
$$

Now $L_{p}=\mu_{0} r_{\rho}\left[\ln \left(8 r_{\rho} / r_{0}\right)-2\right]$, where $\mu_{0}=4 \pi \times 10^{-7} \mathrm{H} / \mathrm{m}$ and $r_{0}$ is the radius of the wire [55]. For a reasonable range of values of $r_{p} / r_{0}$ we can set $L_{p} \approx 5 \mu_{0} r_{p}$. Thus we obtain $B_{N}^{(p)} \approx 2\left(\mu_{0} \epsilon\right)^{1 / 2} / \alpha r_{p}^{3 / 2}$. This indicates that one can, in principle, improve the magnetic field resolution indefinitely by increasing $r_{p}$, keeping $L_{i}=L_{p}$. Of course, in practice, the size of the cryostat will impose an upper limit on $r_{p}$. If we take $\epsilon=10^{-28} \mathrm{~J} \cdot \mathrm{Hz}^{-1}$ (a somewhat conservative value for an RF SQUID), $\alpha=1$, and $r_{p}=25 \mathrm{~mm}$, we find $B_{N}^{(p)} \approx 5 \times 10^{-15}$ $\mathrm{T} \cdot \mathrm{Hz}^{-1 / 2}=5 \times 10^{-11} \mathrm{G} \cdot \mathrm{Hz}^{-1 / 2}$. This is a much higher sensitivity than that achieved by any nonsuperconducting magnetometer.

Magnetometers have usually involved flux transformers made of $\mathrm{Nb}$ wire. For example, one can make the RF SQUID in Fig. 9 into a magnetometer merely by connecting a loop of $\mathrm{Nb}$ wire to its input terminals. In the case of the thin-film dc SQUID, one can make an integrated magnetometer by fabricating an $\mathrm{Nb}$ loop across the spiral input coil. In this way, Wellstood et al. [14] achieved a magnetic field white noise of $5 \times 10^{-15} \mathrm{~T} \cdot \mathrm{Hz}^{-1 / 2}$ with a pickup loop a few millimeters across.

Magnetometers with typical sensitivities of $0.01 \mathrm{pT}$. $\mathrm{Hz}^{-1 / 2}$ have been used in geophysics in a variety of appli- cations, ${ }^{2}$ such as magnetotellurics, active electromagnetic sounding, piezomagnetism, tectonomagnetism, and the location of hydrofractures. Although SQUID-based magnetometers are substantially more sensitive than any other type, the need to replenish the liquid helium in the field has restricted the extent of their applications. For this reason, the advent of high-temperature superconductors may have considerable impact on this field (see Section V).

An important variation of the flux transformer is the gradiometer. Fig. 10(b) shows an axial gradiometer that measures $\partial B_{z} / \partial z$. The two pickup loops are wound in opposition and balanced so that a uniform field $B_{z}$ links zero net flux to the transformer. A gradient $\partial B_{z} / \partial z$, on the other hand, does induce a net flux and thus generates an output from the flux-locked SQUID. Fig. 10(c) illustrates a second-order gradiometer that measures $\partial^{2} B_{z} / \partial z^{2} ;$ Fig. 11(a) shows a practical version.

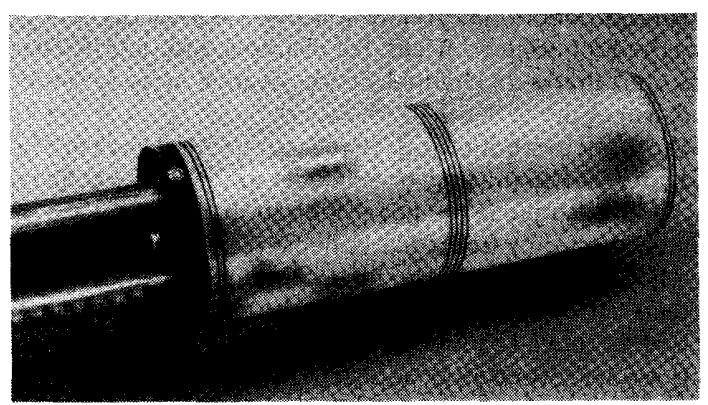

(a)

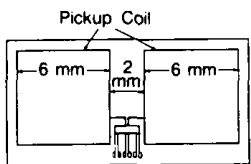

(b)

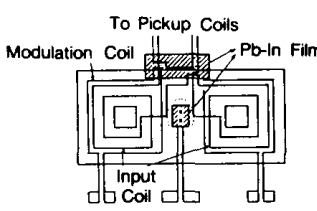

(c)

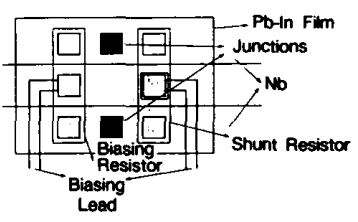

(d)
Fig. 11. (a) Wire-wound second-derivative gradiometer for biomedical applications. (Courtesy of BTi, Inc.) (b) Pickup loops of thin-film first-derivative gradiometer. (c) Two-hole SQUID with spiral input coils. (d) Expanded view of dotted circle in (c), showing junctions and resistive shunts. (From [60].)

Thin-film gradiometers based on dc SQUIDs were made as long ago as 1978 [24], and a variety of devices have been reported since [21], [57]-[61]. To our knowledge, all of the gradiometers made to date have been planar, and therefore measure an off-diagonal gradient, for example, $\partial B_{z} / \partial x$ or $\partial^{2} B_{z} / \partial x \partial y$. The configuration of one such device is shown in Fig. 11(b)-(d).

${ }^{2}$ For a review, see [56] 
The most important application of the gradiometer is in neuromagnetism, ${ }^{3}$ notably to detect weak magnetic signals emanating from the human brain. The gradiometer discriminates strongly against distant noise sources, which have a small gradient, in favor of locally generated signals. One can thus use a second-order gradiometer in an unshielded environment, although the present trend is toward using first-order gradiometers in a shielded room of aluminum and Mumetal that greatly attenuates the ambient magnetic noise. In this application, axial gradiometers of the type shown in Fig. 10(a) actually sense magnetic field, rather than the gradient, because the distance from the signal source to the pickup loop is less than the baseline of the gradiometer. The magnetic field sensitivity referred to one pickup loop is typically $10 \mathrm{fT} \cdot \mathrm{Hz}^{-1 / 2}$. Although great progress in this field has been made in recent years, it is generally agreed that one needs an array of 50 to 100 gradiometers to make a clinically viable system. This requirement has greatly spurred the development of integrated thin-film devices. For example, Knuutila [63] has reported that a 24-channel first-derivative array is under construction.

There are two basic kinds of measurements made on the human brain. In the first, one detects spontaneous activity. A classic example is the generation of magnetic pulses by subjects suffering from focal epilepsy [64]. The second kind involves evoked response; for example, Romani et al. [65] detected the magnetic signal from the auditory cortex generated by tones of different frequencies.

There are several other applications of gradiometers. One kind of magnetic monopole detector [66] consists of a gradiometer. The passage of a monopole would link a flux $h / e$ in the pickup loop and produce a step-function response from the SQUID. Gradiometers have recently been of interest in studies of corrosion and in the location of fractures in pipelines and other structures.

\section{B. Susceptometers}

In principle, one can easily use the first-derivative gradiometer of Fig. 10(b) to measure magnetic susceptibility $\chi$. One establishes a static field along the $z$ axis and lowers the sample into one of the pickup loops. Provided $x$ is nonzero, the sample introduces an additional flux into the pickup loop and generates an output from the flux-locked SQUID. A very sophisticated susceptometer is available commercially [67]. A room temperature access enables one to cycle samples rapidly, and one can measure $\chi$ as a function of temperature between 1.8 and $400 \mathrm{~K}$ in fields of up to $5.5 \mathrm{~T}$. The system is capable of resolving a change in magnetic moment as small as $10^{-8}$ EMU.

Novel miniature susceptometers have been developed by Ketchen and coworkers [29], [68], [69]. One version is shown schematically in Fig. 12. The SQUID loop incorporates two pickup loops wound in the opposite sense and connected in series. The two square pickup loops, $17.5 \mu \mathrm{m}$ on a side and with an inductance of about $30 \mathrm{pH}$, are deposited over a hole in the ground plane that minimizes the inductance of the rest of the device. The SQUID is flux biased at the maximum of $V_{\Phi}$ by means of a control current $I_{C}$ in one of the pickup loops. One can apply a magnetic field

${ }^{3}$ For reviews, see [62].

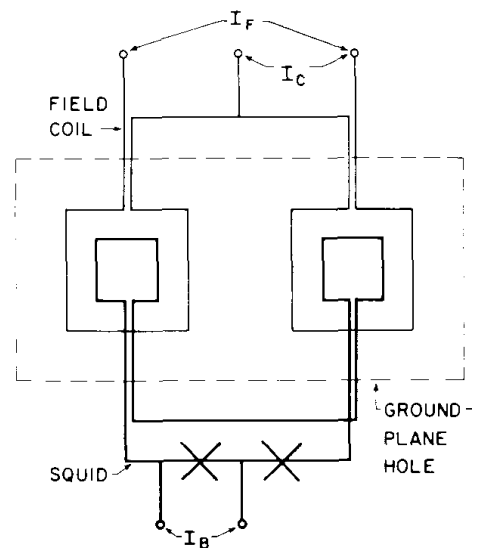

Fig. 12. Thin-film miniature susceptometer. (From [68].)

to the two loops by means of the current $I_{F}$. By passing a fraction of this current into the center $\operatorname{tap} I_{C}$, one can achieve a high degree of electronic balance between the two loops. The sample to be studied is placed over one of the loops, and the output from the SQUID when the field is applied is directly proportional to the magnetization. At $4.2 \mathrm{~K}$, the susceptometer is capable of detecting the magnetization resulting from as few as 3000 electron spins. Awschalom and coworkers [29], [69] have used a miniature susceptometer to perform magnetic spectroscopy of semiconductors with picosecond time resolution.

\section{Voltmeters}

Probably the first practical application of a SQUID was to measure tiny, quasistatic voltages [70]. One simply connects the signal source-for example, a low resistance through which a current can be passed-in series with a known resistance and the input coil of the SQUID. The output from the flux-locked loop is connected across the known resistance to obtain a null-balancing measurement of the voltage. The resolution is generally limited by $\mathrm{Ny}$ quist noise in the input circuit, which at $4.2 \mathrm{~K}$ varies from about $10^{-15} \mathrm{~V} \cdot \mathrm{Hz}^{-1 / 2}$ for a resistance of $10^{-8} \Omega$ to about $10^{-10} \mathrm{~V} \cdot \mathrm{Hz}^{-1 / 2}$ for a resistance of $100 \Omega$.

Applications of these voltmeters range from the measurement of thermoelectric voltages and of quasiparticle charge imbalance in nonequilibrium superconductors to noise thermometry and the comparison of the Josephson voltage-frequency relation in different superconductors to high precision.

\section{The dc SQUID as an RF Amplifier}

Over recent years, the dC SQUID has been developed into a low-noise amplifier for frequencies up to $100 \mathrm{MHz}$ or more [71]. To understand the theory for the performance of this amplifier, we need to extend the theory of Section II by taking into account the noise in the current $J(t)$ in the SQUID loop. For a bare SQUID with $\beta=1, \Gamma=0.05$, and $\Phi=(2 n+1) \Phi_{0} / 4$ one finds the spectral density of the current to be [72]

$$
S_{f}(f) \approx \frac{11 k_{B} T}{R}
$$


Furthermore, the current noise is partially correlated with the voltage noise across the SQUID, the cross-spectral density being [72]

$$
S_{V J}(f) \approx 12 k_{B} T .
$$

The correlation arises, roughly speaking, because the current noise generates a flux noise which in turn contributes to the total voltage noise across the junction provided $V_{\Phi}$ $\neq 0$.

One can make a tuned amplifier, for example, by connecting an input circuit to the SQUID, as shown in Fig. 13.

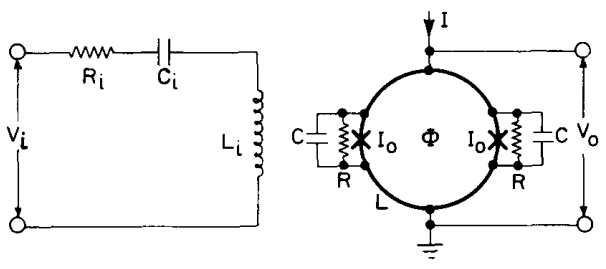

Fig. 13. Tuned RF amplifier based on dc SQUID. (From [71].)

In general, the presence of this circuit modifies all of the SQUID parameters and the magnitude of the noise spectral densities [73]. Furthermore, the SQUID reflects an impedance $\omega^{2} M_{i}^{2} / Z$ into the input circuit [74], where $Z$ is the dynamic input impedance of the SQUID. Fortunately, however, one can neglect the mutual influence of the SQUID and the input circuit provided the coupling coefficient $\alpha^{2}$ is sufficiently small, as it is under certain circumstances. For a signal at frequency $f$ generated by a source with resistance $R_{i}$ one can optimize the noise temperature of the amplifier using standard procedures to obtain

$$
T_{N}^{\mathrm{opt}}=\frac{\pi f}{k_{B} V_{\Phi}}\left(S_{V} S_{J}-S_{V J}^{2}\right)^{1 / 2} .
$$

This minimum value actually occurs off resonance. If one wishes to operate the amplifier at the resonant frequency of the input circuit, the noise temperature is increased to

$$
T_{N}^{\text {res }}=\frac{\pi f}{k_{B} V_{\Phi}}\left(S_{V} S_{\jmath}\right)^{1 / 2}
$$

The corresponding power gain is

$$
G \approx \frac{V_{\Phi}}{\omega} .
$$

Equations (30) and (31) can be shown to imply $\alpha^{2} Q \approx 1$, that is, the coupling is weak for a high-Q input circuit. We emphasize that the noise temperatures quoted in Eqs. (29) and (30) do not include the Nyquist noise generated in $R_{i}$ which may well exceed the noise generated by the amplifier. Thus, Eq. (29) or Eq. (30) does not necessarily represent the lowest system noise temperature, which may well occur under quite different conditions. Nonetheless, these expressions are very useful in that they specify the performance at a particular frequency in terms of the SQUID parameters only.

Hilbert and Clarke [71] made several RF amplifiers with both tuned and untuned inputs, flux biasing the SQUID near $\Phi=(2 n+1) \Phi_{0} / 4$. There was no flux-locked loop. The measured parameters were in good agreement with pre- dictions. For example, for an amplifier with $R \approx 8 \Omega, L \approx$ $0.4 \mathrm{nH}, L_{i} \approx 5.6 \mathrm{nH}, M_{i} \approx 1 \mathrm{nH}$, and $V_{\Phi} \approx 3 \times 10^{10} \mathrm{~s}^{-1}$ at $4.2 \mathrm{~K}$ they found $G=18.6 \pm 0.5 \mathrm{~dB}$ and $T_{N}=1.7 \pm 0.5 \mathrm{~K}$ at $93 \mathrm{MHz}$. The predicted values were $17 \mathrm{~dB}$ and $1.1 \mathrm{~K}$, respectively.

To conclude this discussion, we comment briefly on the quantum limit of the dc SQUID amplifier. At $T=0$, Nyquist noise in the shunt resistors in Eqs. (7) and (8) should be replaced with zero point fluctuations. Koch et al. [75] performed a simulation in this limit and concluded that, within the limits of error, the noise temperature of a tuned amplifier in the quantum limit should be given by

$$
T_{N} \approx \frac{h f}{k_{B} \ln 2} .
$$

This is the result for any quantum limited amplifier. The corresponding value for $\epsilon$ was approximately $\hbar$, but it should be emphasized that quantum mechanics does not impose any precise lower limit on $\epsilon$ [76]. A number of SQUIDs have obtained noise energies of $3 \hbar$ or less, but there is no evidence as yet that a SQUID has attained quantum limited performance as an amplifier.

Clarke, Hahn, and coworkers [77]-[80] have used tuned SQUID amplifiers in a series of experiments to observe nuclear magnetic resonance (NMR) and nuclear quadruple resonance (NQR) at about $30 \mathrm{MHz}$. As an example of the high sensitivity offered by these techniques, they were able to detect "spin noise" in ${ }^{35} \mathrm{Cl}$ nuclei in $\mathrm{NaClO}_{3}$. In zero magnetic field, this nucleus has two doubly degenerate nuclear levels with a splitting of $30.6856 \mathrm{MHz}$ and exhibits NQR. An $R F$ signal at the NQR frequency equalized the populations of the two nuclear spin levels and was then turned off to leave a zero-spin state. A SQUID amplifier was able to detect the photons emitted spontaneously as the upper state decayed, even though the lifetime per nucleus against this process was $\sim 10^{6}$ centuries. The detected power was about $5 \times 10^{-21} \mathrm{~W}$ in a bandwidth of about $1.3 \mathrm{kHz}$.

\section{E. Gravity Wave Antennas}

A quite different application of SQUIDs is to detect minute displacements, notably those of Weber bar gravity wave antennas. ${ }^{4,5}$ Roughly a dozen groups worldwide are using these antennas to search for the pulse of gravitational radiation that is expected to be emitted when a star collapses. The radiation induces longitudinal oscillations in the large, freely suspended bar, but because the amplitude is very tiny, one requires the sensitivity of a dc SQUID to detect it. As an example, we briefly describe the antenna at Stanford University, which consists of an aluminum bar $3 \mathrm{~m}$ long, weighing $4800 \mathrm{~kg}$, suspended in a vacuum chamber at 4.2 $K$. The fundamental longitudinal mode is at $\omega_{2} / 2 \pi \approx 842 \mathrm{~Hz}$, and the $Q$ is $5 \times 10^{6}$. The transducer is shown schematically in Fig. 14. A circular Nb diaphragm is clamped at its perimeter to one end of the bar, with a flat spiral coil made of $\mathrm{Nb}$ wire mounted on each side. The two coils are connected in parallel with each other and with the input coil of a SQUID. This entire circuit is superconducting. A persistent supercurrent circulates in the closed loop formed by the two spiral coils. The associated magnetic fields exert a re-

${ }^{4}$ For an elementary review on gravity waves, see [81].

${ }^{5}$ For a review on gravity wave antennas, see [82]. 


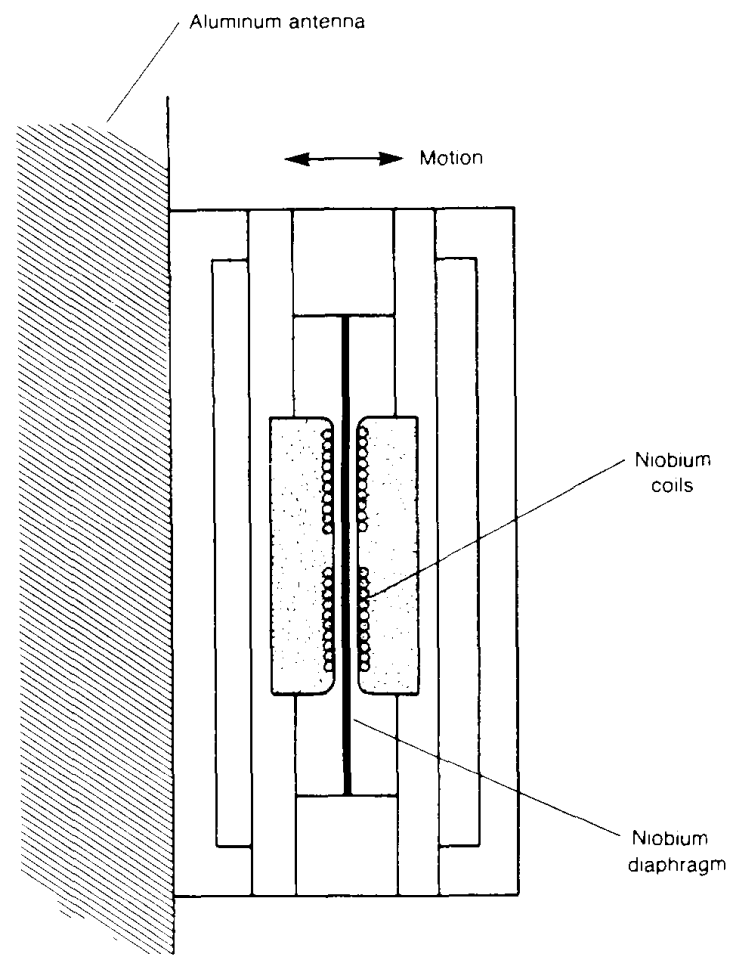

Fig. 14. Transducer for gravity wave antenna. (Courtesy of P. F. Michelson.)

storing force on the diaphragm so that by adjusting the current, one can set the resonant frequency of the diaphragm equal to that of the bar. A longitudinal oscillation of the bar induces an oscillation in the position of the diaphragm relative to the two coils, thereby modulating their inductances. As a result of flux quantization, a fraction of the stored supercurrent is diverted into the input coil of the SQUID, which detects it in the usual way.

The present Stanford antenna has a root-mean-square strain sensitivity $\left\langle(\delta l)^{2}\right\rangle^{1 / 2} / /$ of $10^{-18}$, where $/$ is the length of the bar and $\delta l$ its longitudinal displacement. This very impressive sensitivity, which is limited by thermal noise in the bar, is nonetheless adequate only to detect events in our own galaxy. Because such events are rare, there is a very strong motivation to make major improvements in the sensitivity.

If the bar could be cooled sufficiently, the strain resolution would be limited only by the bar's zero-point motion and would have a value of about $3 \times 10^{-21}$. At first sight one might expect that the bar would have to be cooled to an absurdly low temperature to achieve this quantum limit, because a frequency of $842 \mathrm{~Hz}$ corresponds to a temperature $h \omega_{a} / k_{B}$ of about $40 \mathrm{nK}$. However, it turns out that one can make the effective noise temperature $T_{\mathrm{e}+f}$ of the antenna much lower than the temperature $T$ of the bar. If a gravitational signal in the form of a pulse of length $\tau_{\mathrm{s}}$ interacts with an antenna that has a decay time $Q / \omega_{i \prime \prime}$ then the effective noise temperature is given approximately by the product of the bar temperature and the pulse length divided by the decay time: $I_{\mathrm{eif}} \approx \tau_{\mathrm{s}} \omega_{\mathrm{a}} T / Q$. Thus one can make the effective noise temperature much less than the temperature of the bar by increasing the bar's resonant quality factor suf- ficiently. To achieve the quantum limit, in which the bar energy $h \omega_{a}$ is greater than the effective thermal energy $k_{B} T_{\text {eit }}$, one would have to lower the temperature $T$ below $Q h / k_{B} \tau_{s}$, which is about $40 \mathrm{mK}$ for a quality factor $Q$ of $5 \times 10^{6}$ and a pulse length $\tau_{\mathrm{s}}$ of $1 \mathrm{~ms}$. One can cool the antenna to this temperature with the aid of a large dilution refrigerator.

Needless to say, to detect the motion of a quantum-limited antenna one needs a quantum-limited transducer, a requirement that has been the major driving force in the development of ultra-low-noise dc SQUIDs. As we have seen, however, existing dc SQUIDs at low temperatures are now within striking distance of the quantum limit, and there is every reason to believe that one will be able to operate an antenna quite close to the quantum limit within a few years.

\section{F. Gravity Gradiometers}

The gravity gradiometer, which also makes use of a transducer to detect minute displacements, has been pioneered by Paik [83] and Mapoles [84]. The gradiometer consists of two $\mathrm{Nb}$ proof masses, each constrained by springs to move along a common axis (Fig. 15). A single-layer spiral coil of

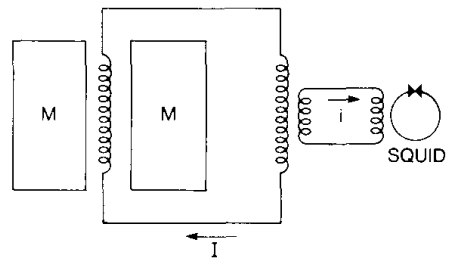

Fig. 15. Gravity gradiometer showing two proof masses (M) on either side of planar spiral coil. (From [84].)

$\mathrm{Nb}$ wire is attached to the surface of one of the masses so that the surface of the wire is very close to the opposing surface of the other mass. Thus, the inductance of the coil depends on the separation of the two proof masses, which in turn depends on the gravity gradient. The coil is connected to a second superconducting coil, which is coupled to a SQUID via a superconducting transformer. A persistent supercurrent I maintains a constant flux in the detector circuit. Thus, a change in the inductance of the pickup coil produces a change in I and hence a flux in the SQUID that is related to the gravity gradient. More sophisticated versions of this design enable one to balance the restoring forces of the two springs electronically [84], thereby eliminating the response to an acceleration (as opposed to an acceleration gradient). Sensitivities of a few Eötvös . $\mathrm{Hz}^{-1 / 2}$ have been achieved at frequencies above $2 \mathrm{~Hz}$ (1 Eötvös $=10^{-9} \mathrm{~s}^{-2}$ ).

Instruments of this kind could be used to map the earth's gravity gradient and have potential in tests of the inverse square law and in inertia! navigation.

\section{The Impaci of High-Temperaturf Superconductivity}

The advent of the high-transition-temperature $\left(T_{C}\right)$ superconductors [85] has stimulated great interest in the prospects for superconducting devices operating at liquid nitrogen (LN) temperatures ( $77 \mathrm{~K}$ ). Indeed, a number of groups have already operated SQUIDs successfully. In this section a brief overview of this work is given. 


\section{A. Predictions for White Noise}

In designing a SQUID for operation at $\mathrm{LN}$ temperatures one must bear in mind the constraints imposed by thermal noise on the critical current and inductance, Eqs. (3) and (4). For $T=77 \mathrm{~K}$, we find $I_{0}>16 \mu \mathrm{A}$ and $L<0.8 \mathrm{nH}$. If we take as arbitrary but reasonable values $L=0.2 \mathrm{nH}$ and $I_{0}=20$ $\mu \mathrm{A}$, we obtain $2 L I_{0} / \Phi_{0}=4$ for the dc SQUID and $L I_{0} / \Phi_{0}=2$ for the RF SQUID. These values are not too far removed from optimum, and to a first approximation we can use the equations for the noise energy given in Sections II and III.

For the case of the dc SQUID, the noise energy is predicted by either Eq. (11) or (12). However, since nobody has yet made a Josephson tunnel junction with high- $T_{C}$ materials, it is somewhat unrealistic to use Eq. (12), which involves the junction capacitance, and instead we use Eq. (11). The value of $R$ is an open question, and we rather arbitrarily adopt $5 \Omega$, which is not too different from values achieved experimentally for high- $T_{C}$ grain boundary junctions [86]. With $L=0.2 \mathrm{nH}, T=77 \mathrm{~K}$, and $R=5 \Omega$, Eq. (11) predicts $\epsilon$ $\approx 4 \times 10^{-31} \mathrm{~J} \cdot \mathrm{Hz}^{-1}$. This value is only about one order of magnitude higher than that found at $4.2 \mathrm{~K}$ for typical $\mathrm{Nb}$ based thin-film dc SQUIDs, and actually somewhat better than that found in commercially available toroidal SQUIDs. These various values are summarized in Fig. 16. If one could

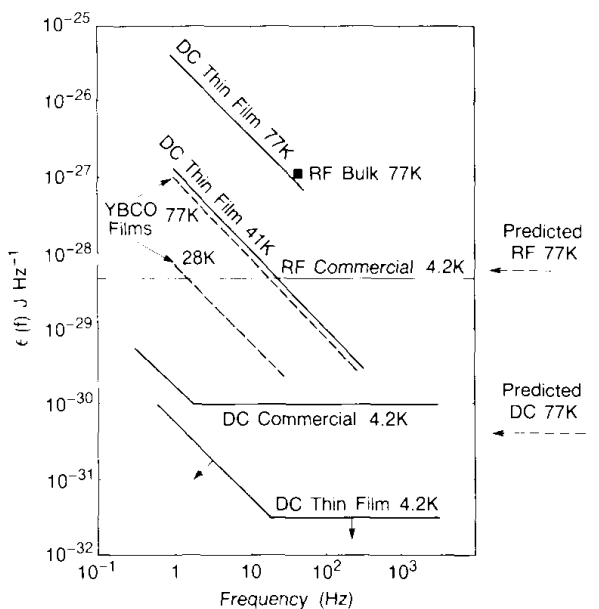

Fig. 16. Noise energy $\epsilon(f)$ versus frequency for several SQUIDs and for a YBCO film.

actually achieve the predicted resolution in a SQUID at 77 $\mathrm{K}$ at frequencies down to $1 \mathrm{~Hz}$ or less, it would be adequate for most of the applications discussed in Section IV.

For the RF SQUID, Eq. (18) predicts an intrinsic noise energy of about $6 \times 10^{-29} \mathrm{~J} \cdot \mathrm{Hz}^{-1}$ for $I_{0}=20 \mu \mathrm{A}, L I_{0}=2 \Phi_{0}$, $\omega_{\mathrm{RF}} / 2 \pi=20 \mathrm{MHz}$, and $T=77 \mathrm{~K}$. This value is comparable with the overall value obtained experimentally with $4.2 \mathrm{~K}$ devices where the effective noise temperature $T_{a}^{\text {eff }}$ of the preamplifier and tank circuit is much higher than the bath temperature when the preamplifier is at room temperature (see Section III-B). However, when one operates a SQUID at $77 \mathrm{~K}$, there is no reason for $T_{a}^{\mathrm{eff}}$ to increase, and the system noise energy should be comparable with that at $4.2 \mathrm{~K}$.

With regard to $1 /$ fnoise, in general one might expect both critical current noise and flux noise to contribute. However, it seems impractical to make any a priori predictions of the magnitude of these contributions.

\section{B. Practical Devices}

Although a number of $d c$ and RF SQUIDs have been made from $\mathrm{YBa}_{2} \mathrm{Cu}_{3} \mathrm{O}_{7-\delta}$ ( $\mathrm{YBCO}$ ), just one of each type is described here. It appears that the first dc SQUID was made by Koch et al. [86]. In their first devices, they patterned the films by covering the regions of YBCO to remain superconducting with a gold film, and ion implanted the unprotected regions so that they became insulators at low temperatures. The configuration is shown in Fig. 17; the

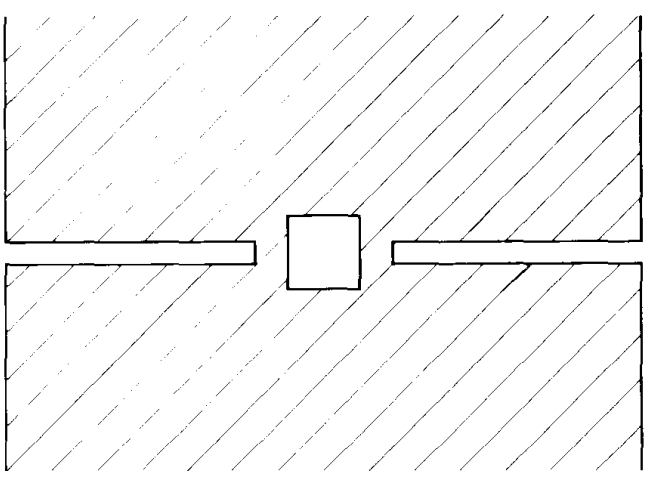

Fig. 17. Planar thin-film dc SQUID fabricated from YBCO. (Redrawn from [86].)

estimated inductance is $80 \mathrm{pH}$. The two microbridges exhibited Josephson-like behavior, which actually arose from junctions formed by grain boundaries between randomly oriented grains of YBCO. As the quality of the films has improved, conventional patterning techniques such as liftoff and ion etching have become possible. The $1-V$ characteristics of these devices are modulated by an applied flux, although the $V-\Phi$ curves are often hysteretic and nonperiodic, probably because of flux trapped in the YBCO films. The noise energy scaled approximately as $1 /$ fover the frequency range investigated, usually 1 to $10^{3} \mathrm{~Hz}$. The lowest noise energies achieved to date at $1 \mathrm{~Hz}$ are $4 \times 10^{-27} \mathrm{~J}$ - $\mathrm{Hz}^{-1}$ at $41 \mathrm{~K}$ and, in a different device, $2 \times 10^{-26} \mathrm{~J} \cdot \mathrm{Hz}^{-1}$ at $77 \mathrm{~K}$. These values are plotted in Fig. 16.

The best characterized RF SQUID reported so far is that of Zimmerman et al. [87]. They drilled a hole along the axis of a cylindrical pellet of YBCO and cut a slot partway along a radius (Fig. 18). The pellet was glued into an aluminum holder, also with a slot, and the assembly immersed in LN. A taper pin forced into the slot in the mount caused the YBCO to break in the region of the cut. When the pin was withdrawn slightly, the YBCO surfaces on the two sides of the crack were brought together, forming a "break junction." The RF SQUID so formed was coupled to a resonant circuit and operated in the usual way. The best flux resolution was $4.5 \times 10^{-4} \Phi_{0} \mathrm{~Hz}^{-1 / 2}$ at $50 \mathrm{~Hz}$, corresponding to a noise energy of $1.6 \times 10^{-27} \mathrm{~J} \cdot \mathrm{Hz}^{-1}$ for $L=0.25 \mathrm{nH}$ (see Fig. 16).

\section{Flux Noise in YBCO Films}

It is evident that the $1 / f$ noise level in YBCO SQUIDs is very high compared with that in $\mathrm{Nb}$ or $\mathrm{Pb}$ devices at $4.2 \mathrm{~K}$. Ferrari et al. [88] have investigated the source of this noise by measuring the flux noise in YBCO films. Each film, deposited on $\mathrm{SrTiO}_{3}$ chip, was patterned into a loop and 


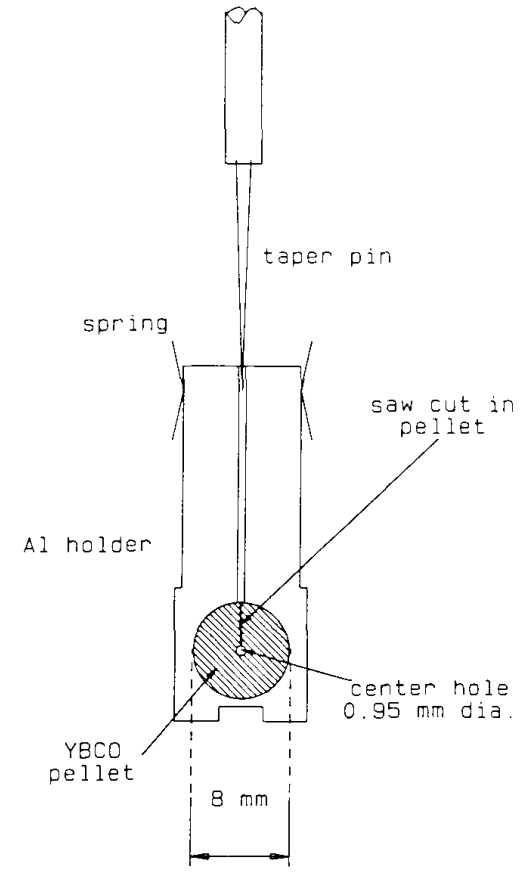

Fig. 18. Break-junction RF SQUID. (From [87].)

mounted parallel and very close to an Nb-based SQUID (with no input coil) so that any flux noise in the YBCO loop could be detected by the SQUID. The assembly was enclosed in a vacuum can immersed in liquid helium. The SQUID was maintained at $4.2 \mathrm{~K}$, while the temperature of the $\mathrm{YBCO}$ film could be increased by means of a resistive heater. Below $T_{C}$, the spectral density of the flux noise scaled as $1 /$ fover the frequency range of 1 to $10^{3} \mathrm{~Hz}$, and increased markedly with temperature. Three films were studied, with microstructure improving progressively with respect to the fraction of grains oriented with the $c$ axis perpendicular to the substrate. The critical current density correspondingly increased, to a value of $2 \times 10^{6} \mathrm{~A} \cdot \mathrm{cm}^{-2}$ at $4.2 \mathrm{~K}$ in the best film. The spectral density of the noise measured at $1 \mathrm{~Hz}$ is shown versus temperature in Fig. 19. We see that in each

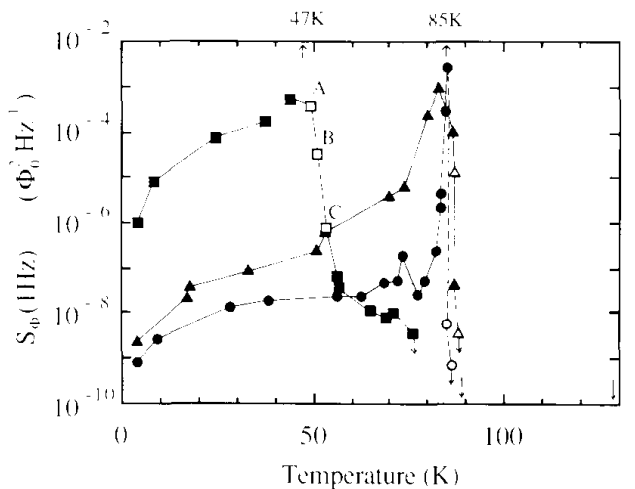

Fig. 19. Spectral density of flux noise at $1 \mathrm{~Hz}$ versus tem. perature for three YBCO films. I - polycrystalline; $\triangle-$ mixed $a$ and $\mathrm{c}$ axes; ... $->90 \% \mathrm{c}$ axis. Solid symbols - noise is $1 / f$ at $1 \mathrm{~Hz}$; open symbols - white or nearly white noise. (From [88].) case the noise increases rapidly as the temperature approaches $T_{C}$, and that, at a given temperature, the noise decreases dramatically as the quality of the films is improved. The noise energy estimated at 28 and $77 \mathrm{~K}$ with an assumed inductance of $400 \mathrm{pH}$ is shown in Fig. 16.

These results demonstrate that $\mathrm{YBCO}$ films are intrinsically noisy. The noise presumably arises from the motion of flux quanta trapped in the films, possibly at grain boundaries. This mechanism is almost certainly the origin of the $1 / f$ noise observed in YBCO SQUIDs and, in general terms, is similar to the origin of $1 / f$ noise in Nb SQUIDs. It is encouraging that the noise is reduced as the microstructure of the films is improved, and it should be emphasized that there is no reason to believe the lowest noise measured so far represents a lower bound. The implication is that SQUIDs and flux transformers coupled to them should be made of very high-quality films.

\section{Future Prospects for High-T $\mathrm{T}_{\mathrm{C}}$ SQUIDs}

One potential application of a high- $T_{C}$ SQUID is as a geophysical magnetometer (see Section IV-A). At the moment, however, it is not entirely straightforward to predict the performance, since the devices are still evolving and it is evident from the noise measurements on YBCO films that thinfilm flux transformers may introduce considerable levels of low-frequency noise. To make some kind of estimate, we assume that we can optimally couple the $77 \mathrm{~K}$ dc SQUID with the noise energy shown in Fig. 16 to a noiseless flux transformer with a thin-film pickup loop with a diameter of $50 \mathrm{~mm}$. The estimated loop inductance is about $150 \mathrm{nH}$. Using Eq. (26), we find a magnetic field resolution of roughly $0.1 \mathrm{pT} \cdot \mathrm{Hz}^{-1 / 2}$ at $1 \mathrm{~Hz}$, improving to $0.01 \mathrm{pT} \cdot \mathrm{Hz}^{-1 / 2}$ at 100 $\mathrm{Hz}$. Although this performance is quite good, one should realize that commercially available coils operated at room temperature offer a resolution of about $0.03 \mathrm{pT} \cdot \mathrm{Hz}^{-1 / 2}$ over this frequency range. Furthermore, our assumption of a noise-free flux transformer is rather optimistic. Nonetheless, given the short time over which the high- $T_{C}$ materials have been available one should be rather encouraged. A relatively modest reduction in the $1 / f$ noise that might be gained from improving the quality of $\mathrm{YBCO}$ films or even from using alternative materials might well yield a rather useful geophysical device.

One might note here that the real advantage in using liquid nitrogen as opposed to liquid helium for field applications is not really the reduction in cost, a savings that is negligible compared with the cost of mounting a field operation, but rather the very much slower boil-off rate of liquid nitrogen. The latent heat of vaporization of liquid $\mathrm{N}_{2}$ is about 60 times that of liquid ${ }^{4} \mathrm{He}$, so that one should be able to design cryostats of modest size with hold times of up to a year. It is also noteworthy that liquid $\mathrm{Ne}$, which boils at 28 $\mathrm{K}$, has a latent heat roughly 40 times that of liquid $\mathrm{He}$, and its use would also greatly extend the running time over that of liquid $\mathrm{He}$, for roughly the same cost per day. We see from Fig. 19 that the $1 / f$ noise in YBCO films can be considerably less at $28 \mathrm{~K}$ than at $77 \mathrm{~K}$, so that the lower temperature operation could be a considerable advantage.

The likely impact of high- $T_{C}$ SQUIDs on the more demanding applications such as neuromagnetism is much smaller, however, at least for the near future. Here one needs very high sensitivity at frequencies down to $0.1 \mathrm{~Hz}$ 
or less, but is not particularly concerned with the cost of liquid ${ }^{4} \mathrm{He}$ or the need to replenish it every day or two. Furthermore, low-noise, closed-cycle refrigerators are just becoming available, which obviate the need to supply liquid cryogens in environments where electric power is available. Thus it is difficult to imagine that high- $T_{C}$ SQUIDs will have a significant impact in this area unless there is a major reduction in the $1 / f$ noise.

In concluding this section, we note that two key problems must be solved before high- $T_{C}$ SQUIDs are likely to become technologically important. The first is the development of a reproducible and reliable josephson junction. Although great progress has been made with grain boundary junctions, it is not clear that one can base a technology on this technique. Shiota et al. [89] have reported $Y B C O-$ insulator-YBCO junctions formed by fluorination of the base electrode, but the $I-V$ characteristics revealed that one of the surfaces was normal. Hopefully, it will be possible to produce all-YBCO junctions exhibiting Josephson tunneling in the near future. An alternative might be a superconductor-normal-metal-superconductor junction [90]. The second problem is concerned with the reduction of hysteresis and noise in thin films of high- $T_{C}$ material. The motion of magnetic flux in the films is responsible for both effects, and one has to learn to produce films with lower densities of flux lines or higher pinning energies. Given the worldwide effort on the new superconductors, there is every reason to be optimistic about the long-term future of SQUIDs based on these materials.

\section{Concluding Rrmarks}

We have tried to give an overview of the current status of $\mathrm{dc}$ and RF SQUIDs. We make no pretense that this account is comprehensive. Many SQUID designs and applications were not mentioned, but we hope to have given some flavor of the amazing versatility of these devices. For example, we find it remarkable that a dc SQUID is the basis of both the most sensitive magnetometer available at $10^{-4}$ $\mathrm{Hz}$ and the quietest RF amplifier at $10^{8} \mathrm{~Hz}$.

The RF SQUID remains more widely used than the dc SQUID, simply because it is produced commercially and is thus available to people who are interested in using SQUIDs rather than in making them. One should also realize that although the kind of RF SQUID routinely used is much less sensitive than state-of-the-art thin-film dc SQUIDs, it is nonetheless entirely adequate for many applications. The fact that thin-film integrated dc SQUIDs are not available commercially is presumably because the cost of establishing a facility to produce them is high while the perceived market is small. However, this situation may be about to change. After all, one needs only a single major application to make reasonably priced SQUIDs available for any number of applications, and there now seem to be two such major products on the horizon. The first is in neuromagnetism. If this application is to become a clinical reality, one will need systems with as many as 100 channels, and the need for 100 channels will inevitably lead to the production of thin-film SQUIDs on a reasonable scale. The second is the advent of high- $T_{C}$ thin-film SQUIDs. If these devices attain sufficient sensitivity and reliability for geophysical applications, not to mention laboratory-based applications such as voltmeters, they will be in sufficient demand to justify production on a commercial basis.

\section{Note Added in Proof}

Recently Koch et al. [91] operated dc SQUIDs fabricated from $\mathrm{TIBaCaCuO}$ at $77 \mathrm{~K}$ and found that a few showed an appreciably lower noise level than YBCO devices. At low frequencies, the spectral density of the noise was $1 / f$. At 10 $\mathrm{Hz}$, the best values of the noise energy were $5 \times 10^{-29}$ and $2 \times 10^{-29} \mathrm{~J} \cdot \mathrm{Hz}^{-1}$ for devices with inductances of about 80 and $5 \mathrm{pH}$, respectively. The low-frequency noise was neither the flux noise nor the critical noise described in Section II-E, but appeared to arise from the rearrangements of flux vortices pinned in the microbridges. The low level of flux noise was possibly due to the large grain sizes of the TIBaCaCuO films $(10-40 \mu \mathrm{m})$ compared with those of the YBCO films $(-1 \mu \mathrm{m})$. Most of the Tl-based SQUIDs were approximately in the configuration of Fig. 17 and exhibited $V-\Phi$ curves that were rather aperiodic and hysteretic. Fortunately, when the devices were operated in a flux-locked loop (Section II-C), the flux in the SQUID was kept constant and the hysteresis had little or no effect on the performance. Subsequently, a device fabricated with a narrow loop showed periodic $V-\Phi$ curves with neg!igible hysteresis [92].

Although these TI-based SQUIDs relied on grain boundary junctions, their noise level would be low enough for many applications if a suitable low-noise flux transformer could be made. The outlook for high-TC SQUIDs seems to be extremely promising.

\section{ACKNOWLEDGMENI}

The author is indebted to D. Crum for supplying Fig. 11(a), to P. F. Michelson for Fig. 14, to D. Paulsen for Fig. 9, to F. C. Wellstood for Fig. 6, and to J. E. Zimmerman for Fig. 18. He wishes to thank R. H. Koch and F. C. Wellstood for very helpful conversations.

\section{ReFERENCES}

[1] F. London, Superfluids. New York, NY: Wiley, 1950.

[2] B. D. Josephson, "Possible new effects in superconductive tunneling," Phys. Lett., vol. 1, pp. 251-253, 1962; "Supercurrents through barriers," Adv. Phys., vol. 14, pp. 419-451, 1965.

[3] R. C. Jaklevic, I. Lambe, A. H. Silver, and J. E. Mercereau, "Quantum interference effects in Josephson tunneling," Phys. Rev. Lett., vol. 12, pp. 159-160, 1964.

[4] J. E. Zimmerman, P. Thiene, and J. T. Harding, "Design and operation of stable rf-biased superconducting point-contact quantum devices, and a note on the properties of perfectly clean metal contacts," J. Appl. Phys., vol. 41, pp. 1572-1580, 1970.

[5] J. E. Mercereau, "Superconducting magnetometers," Rev. Phys. Appl., vol. 5, pp. 13-20, 1970; M. Nisenoff, "Superconducting magnetometers with sensitivities approaching $10^{-10}$ gauss," Rev. Phys. Appl., vol. 5, pp. 21-24, 1970.

[6] W.C. Stewart, "Current-voltage characteristics of Josephson junctions," Appl. Phys. Lett., vol. 12, pp. 277-280, 1968.

[7] D. E. McCumber, "Effect of ac impedance on dc voltage-current characteristics of Josephson junctions," J. Appl. Phys., vol. 39. pp. 3113-3118, 1968.

[8] J. Clarke and R. H. Koch, "The impact of high-temperature superconductivity on SQUIDs," Science, vol. 242, pp. 217-223, 1988.

[9] C. D. Tesche and J. Clarke, "DC SQUID: noise and optimization," J. Low Temp. Phys., vol. 27, pp. 301-331, 1977.

[10] J. ]. P. Bruines, V. J. de Waal, and J. E. Mooij, "Comment on 'dc SQUID noise and optimization' by Tesche and Clarke," J. Low Temp. Phys., vol. 46, pp. 383-386, 1982.

[11] V.J. de Waal, P. Schrijner, and R. Llurba, "Simulation and opti- 
mization of a dc SQUID with finite capacitance," J. Low Temp. Phys., vol. 54, pp. 215-232, 1984.

[12] M. B. Ketchen and I. M. Jaycox "Ultra-low noise tunnel junction de SQUID with a tightly coupled planar input coil," Appl. Phys. Lett., vol. 40, pp. 736-738, 1982.

[13] J. M. Jaycox and M. B. Ketchen, "Planar coupling scheme for ultra low noise de SQUIDs," IEEF Trans. Magn., vol. MAG-17, pp. 400-403, 1981.

[14] F. C. Wellstood, C. Heiden, and I. Clarke, "Integrated dc SQUID magnetometer with high slew rate," Rev. Sci. Instr. vol. 55 , pp. 952-957, 1984

[15] M. Gurvitch, M. A. Washington, and H. A. Huggins, "High quality refractory losephson tunnel junction utilizing thin aluminum layers," Appl. Phys. Lett., vol. 42, pp. 472-474, 1983.

[16] V.J. de Waal, T. M. Klapwijk, and P. Van den Hamer, "High performance dc SQUIDs with submicrometer niobium Josephson junctions," J. Low Temp. Phys., vol. 53, pp. 287 312, 1983.

[17] C. D. Tesche et al., "Practical dc SQUIDs with extremely low 1/f noise," IEEE Trans. Magn., vol. MAG-21, pp. 1032-1035, 1985.

[18] C. M. Pegrum, D. Hutson, G. B. Donaldson, and A. Tugwell, "DC SQUIDs with planar input coils"" IEEE Trans. Magn., vol. MAG-21, pp. 1036-1039, 1985.

[19] T. Noguchi, N. Ohkawa, and K. Hamanaka, "Tunnel junction dC SQUID with a planar input coil," in SQUID 85 Superconducting Quantum Interference Devices and Their Applications, H. D. Hahlbohm and H. Lubbig, Eds. Berlin, Germany: Walter de Gruyter, 1985, pp. 761-766.

[20] B. Muhlfelder, J.A. Beall, M.W. Cromar, and R.H. Ono, "Very low noise tightly coupled dc SQUID amplifiers," Appl. Phys. Lett., vol. 49, pp. 1118-1120, 1986.

[21] J. Knuutila, N. Kajola, H. Seppä, R. Mutikainen, and I. Salmi, "Design, optimization and construction of a dc SQUID with complete flux transformer circuits," I. Low Temp. Phys., vol. 71, pp. 369-392, 1988.

[22] P. Carelli and V. Foglietti, "Behavior of a multiloop dc superconducting quantum interference device," J. Appl. Phys., vol. 53, pp. 7592-7598, 1982

[23] J. Clarke, W. M. Goubau, and M. B. Ketchen, J. Low Temp. Phys., vol. 25, pp. 99-144, 1976.

[24] M. B. Ketchen, W. M. Goubau, J. Clarke, and G. B. Donaldson, "Superconducting thin-film gradiometer," I. Appl. Phys., vol. 44, pp. 4111-4116, 1978

[25] F. C. Wellstood and J. Clarke, unpublished.

[26] F. C. Wellstood, C. Urbina, and J. Clarke, "Low-frequency noise in dc superconducting quantum interference devices below 1 K," Appl. Phys. Lett., vol. 50, pp. 772-774, 1987.

[27] M. L. Roukes, M. R. Freeman, R. S. Germain, R. C. Richardson, and M. B. Ketchen, "Hot electrons and energy transport in metals at milliketvin temperatures," Phys. Rev. Lett., vol. 55, pp. $422-425,1985$

[28] F. C. Wellstood, C. Urbina, and I. Clarke, "Hot electron effect in the dC SQUID," IEEE Trans. Magn., vol. MAG-25, pp. 10011004, 1989; Appl. Phys. Lett., vol. 54, p. 2599, 1989.

[29] M. B. Ketchen et al., "Design, fabrication and performance of integrated miniature SQUID susceptometers," IEEE Trans. Magn., vol. MAG-25, pp. 1212-1215, 1989.

[30] R. H. Koch et al., "Flicker (1/f) noise in tunnel junction dc SQUIDs," I. Low Temp. Phys., vol. 51, pp. 207-224, 1983.

[31] C. T. Rogers and R. A. Buhrman, "Composition of $1 / f$ noise in metal-insulator-metal tunnel junctions," Phys. Rev. Lett., vol. 53, pp. 1272-1275, 1984.

[32] P. Dutta and P. M. Horn, "Low-frequency fluctuations in solids: 1/f noise," Rev. Mod. Phys., vol. 53, pp. 497-516, 1981.

[33] C. D. Tesche et al., "Well-coupled dc SQUID with extremely low 1/f noise," in Proc. 17th Int. Conf. on Low Temperature Physics, vol. LT-17, pp. 263-264, 1984.

[34] V. Foglietti et al., "Low-frequency noise in low $1 / f$ noise $\mathrm{dc}$ SQUIDs," Appl. Phys. Lett., vol. 49, pp. 1393-1395, 1986.

[35] Biomagnetic Technologies Inc., 4174 Sorrento Valley Blvd., San Diego, CA 92121.

[36] N. Fujimaki, H. Tamura, T. Imamura, and S. Hasuo "A singlechip SQUID magnetometer," in Dig. Tech. Papers of 1988 Int. Solid-State Conf., pp. 40-41, 1988.

[37] D. Drung, "Digital feedback loops for dc SQUIDs," Cryogenics, vol. 26, pp. 623-627, 1986; D. Drung, E. Crocoll, R.
Herwig, M. Neuhaus, and W. Jutzi, "Measured performance parameters of gradiometers with digital output," IEEE Trans. Magn. vol. MAG-25, pp. 1034-1037, 1989.

[38] M. Mück and C. Heiden, "Simple dc SQUID system based on a frequency modulated relaxation oscillator," IEEE Trans. Magn., vol. MAG-25, pp. 1151-1153, 1989.

[39] J. Clarke, "Superconducting quantum interference devices for low frequency measurements," in Superconductor Applications: SQUIDs and Machines, B. B. Schwartz and S. Foner, Eds. New York, NY: Plenum, 1977, pp. 67-124.

[40] R. P. Giffard, R. A. Webb, and J. C. Wheatley, "Principles and methods of low-frequency electric and magnetic measurements using rf-biased point-contact superconducting device," J. Low Temp. Phys., vol. 6. pp. 533-610, 1972.

[41] J. Kurkijärvi, "Intrinsic fluctuations in a superconducting ring closed with a Josephson junction," Phys. Rev. B, vol. 6, pp. 832-835, 1972

[42] J. Kurkijärvi and W. W. Webb, "Thermal noise in a superconducting flux detector," in Proc. Applied Superconductivity Conf., pp. 581-587, 1972.

[43] L. D. Jackel and R. A. Buhrman, "Noise in the rf SQUID," /. Low Temp. Phys., vol. 19, pp. 201-246, 1975.

[44] G. J. Ehnholm, "Complete linear equivalent circuit for the SQUID," in SQUID Superconducting Quantum Interference Devices and Their Applications, H. D. Hahlbohm and H. Lubbig, Eds. Berlin, Germany: Walter de Gruyter, 1977, pp. 485499; "Theory of the signal transfer and noise properties of the rf SQUID," J. Low Temp. Phys., vol. 29, pp. 1-27, 1977.

[45] H. N. Hollenhorst and R. P. Giffard "Input noise in the hys. teretic if SQUID: theory and experiment," I. Appl. Phys., vol. 51, pp. 1719-1725, 1980.

[46] J. Kurkijärvi, "Noise in the superconducting flux detector," J. Appl. Phys., vol. 44, pp. 3729-3733, 1973

[47] R. P. Giffard, ]. C. Gallop, and B. N. Petley, "Applications of the Josephson effects," Prog. Quant. Flectron., vol. 4, pp. 301 $402,1976$.

[48] G. J. Ehnholm, S. T. Islander, P. Ostman, and B. Rantala, "Measurements of SQUID equivalent circuit parameters," coll. C6, J. Physique, vol. 39, pp. 1206-1207, 1978.

[49] R. P. Giffard and J. N. Hollenhorst, "Measurement of forward and reverse signal transfer coefficients for an rf-biased SQUID," Appl. Phys. Lett., vol. 32, pp. 767-769, 1978

[50] A. Long, T. D. Clark, R. J. Prance, and M. G. Richards, "High performance UHF SQUID magnetometer," Rev. Sci. Instr., vol. 50, pp. 1376-1381, 1979.

[51] J. N. Hollenhorst and R. P. Giffard, "High sensitivity microwave SQUID," IEEE Trans. Magn., vol. MAG-15, pp. 474-477, 1979.

[52] J. Clarke, "Advances in SQUID magnetometers," IEFE Trans. Electron Devices, vol. ED-27, pp. 1896-1908, 1980.

[53] H. Ahola, G. H. Ehnholm, B. Rantala, and P. Ostman, "Cryogenic GaAs-FET amplifiers for SQUIDs," coll. C6, 1. Physique, vol. 39, pp. 1184-1185, 1978; I. Low Temp. Phys., vol. 35, pp. $313-328,1979$

[54] J. E. Zimmerman, "Sensitivity enhancement of superconducting quantum interference devices through the use of fractional-turn loops," J. Appl. Phys., vol. 42, pp. 4483-4487, 1971.

[55] D. Shoenberg, Superconductivity. London, England: Cambridge Univ. Press, 1962, p. 30.

[56] J. Clarke, "Geophysical applications of SQUIDs," IEEE Trans. Magn., vol. MAG-19, pp. 288-294, 1983.

[57] V. j. de Waal and T. M. Klapwijk, "Compact integrated dc SQUID gradiometer," Appl. Phys. Lett., vol. 41, pp. 669-671, 1982.

[58] G. J. Van Nieuwenhuyzen and V. J. de Waal, "Second order gradiometer and dC SQUID integrated on a planar substrate," Appl. Phys. Lett., vol. 46, pp. 439-441, 1985.

[59] P. Carelli and V. Foglietti, "A second derivative gradiometer integrated with a dc superconducting interferometer," J. Appl. Phys., vol. 54, pp. 6065-6067, 1983

[60] M. Koyangi, N. Kasaj, K. Chinore, M. Nakanishi, and S. Kosaka, "An integrated dc SQUID gradiometer for biomagnetic application," IEEE Trans. Magn., vol. MAG-25, pp. 1166-1169, 1989.

[61] J. Knuutila, M. Kajola, R. Mutikainen, and J.Salmi, "Integrated planar dc SQUID magnetometers for multichannel neuromagnetic measurements," Proc. ISEC, p. 261, 1987. 
[62] G. L. Romani, S. J. Williamson, and L. Kaufman, "Biomagnetic instrumentation," Rev. Sci. Instr., vol. 53, pp. 1815-1845, 1982; D. S. Buchanan, D. Paulson, and S. J. Williamson, "Instrumentation for clinical applications of neuromagnetism, "Adv. Cryo. Eng., vol. 33, pp. 97-106, 1988.

[63] J. Knuutila, "Multichannel SQUID systems in neuromagnetic research: design and use," presented at the European Physical Society Workshop, SQUID: State of Art, Perspectives and Applications, Rome, Italy, June 22-24, 1988.

[64] D. S. Barth, W. Sutherling, J. Engel, Jr., and J. Beatty, "Neuromagnetic evidence of spatially distributed sources underlying epileptiform spikes in the human brain," Science, vol. 223, pp. 293-296, 1984

[65] G. L. Romani, S. J. Williamson, and L. Kaufman, "Tonotopic organization of the human auditory cortex, "Science, vol. 216 , pp. $1339-1340,1982$.

[66] B. Cabrera, "First results from a superconductive detector for moving magnetic monopoles," Phys. Rev. Lett., vol. 48, pp. 1378-1381, 1982.

[67] Quantum Design, 11568 Sorrento Valley Road, San Diego, CA 92121.

[68] M. B. Ketchen, T. Kopley, and H. Ling, "Miniature SQUID susceptometer," Appl. Phys. Lett., vol. 44, pp. 1008-1010, 1984.

[69] D. D. Awschalom and J. Warnock, "Picosecond magnetic spectroscopy with integrated dc SQUIDs," IEEE Trans. Magn., vol. MAG-25, pp. 1186-1192, 1989.

[70] J. Clarke, "A superconducting galvanometer employing Josephson tunneling," Phil. Mag., vol. 13, pp. 115-127, 1966.

[71] C. Hilbert and J. Clarke, "DC SQUIDs as radiofrequency amplifiers," I. Low Temp. Phys., vol. 61, pp. 263-280, 1985.

[72] C. D. Tesche and I. Clarke, "Dc SQUID: current noise," J. Low Temp. Phys., vol. 37, pp. 397-403, 1979.

[73] J. M. Martinis and I. Clarke. "Signal and noise theory for the dc SQUID," J. Low Temp. Phys., vol. 61, pp. 227-236, 1985, and references therein.

[74] C. Hilbert and J. Clarke, "Measurements of the dynamic input impedance of a dc SQUID," I. Low Temp. Phys., vol. 61, pp. $237-262,1985$.

[75] R. H. Koch, D. J. Van Harlingen, and J. Clarke, "Quantum noise theory for the dc SQUID," Appl. Phys. Lett., vol. 38, pp. 380$382,1981$.

[76] V.V.Danilov, K. K. Likharev, and A. B. Zorin, "Quantum noise in SQUIDs," IEEE Trans. Magn., vol. MAG-19, pp. 572-575, 1983.

[77] C. Hilbert, J. Clarke, T. Sleator, and E. L. Hahn, "Nuclearquadruple resonance detected at $30 \mathrm{MHz}$ with a dc superconducting quantum interference device," Appl. Phys. Lett., vol. 47 , pp. $637-639,1985$, and references therein for earlier work On NMR.

[78] N. Q. Fan et al., "Nuclear magnetic resonance with dc SQUID preamplifiers," IEEE Trans. Magn., vol. MAG-25, pp. 1193-1199, 1989.

[79] T. Sleator, E. L. Hahn, M. B. Heaney, C. Hilbert, and J. Clarke, "Nuclear electric quadrupole induction of atomic polarization," Phys. Rev. Lett., vol. 57, pp. 2756-2759, 1986.

[80] T. Sleator, E. L. Hahn, C. Hilbert, and J. Clarke, "Nuciear-spin noise and spontaneous emission," Phys. Rev. B, vol. 36, pp. 1969-1980, 1987.

[81] S. L. Shapiro, R. F. Stark, and S. J. Teukolsky, "The search for gravitational waves," Am. Sci., vol. 73, pp. 248-257, 1985.
[82] P. F. Michelson, J. C. Price, and R. C. Taber, "Resonant-mass detectors of gravitational radiation," Science, vol. 237, pp. $150-157,1987$

[83] H.l. Paik, "Superconducting tensor gravity gradiometer with SQUID readout," in SQUID Applications to Geophysics, H. Weinstock and W. C. Overton, Jr., Eds. Tulsa, OK: Soc. of Exploration Geophysicists, 1981, pp. 3-12.

[84] E. Mapoles, "A superconducting gravity gradiometer," in SOUID Applications to Geophysics, H. Weinstock and W. C. Overton, Jr., Eds. Tulsa, OK: Soc. of Exploration Geophysicists, 1981, pp. 153-157.

[85] I. G. Bednorz and K. A. Muller, "Possible high $T_{C}$ superconductivity in the Ba-La-Cu-O system," Z. Phys., vol. B64, pp. 189-193, 1986.

[86] R. H. Koch, C. P. Umbach, G. J. Clark, P. Chaudhari, and R. B. Laibowitz, "Quantum interference devices made from superconducting oxide thin films," Appl. Phys. Lett., vol. 51, pp. 200-202, 1987.

[87] J. E. Zimmerman, ]. A. Beall, M. W. Cromar, and R. H. Ono, "Operation of a Y-Ba-Cu-O rf SQUID at $81 \mathrm{~K}, "$ Appl. Phys. Lett., vol. 51, pp. 617-618, 1987.

[88] M. J. Ferrari et al., "Magnetic flux noise in thin film rings of $\mathrm{YBa}_{2} \mathrm{Cu}_{3} \mathrm{O}_{7-\delta}$," Appl. Phys. Lett., vol. 53, pp. 695-697, 1988.

[89] T. Shiota, K. Takechi, Y. Takai, and H. Hayakawa, "An observation of quasiparticle tunneling characteristics in all $\mathrm{Y}$-BaCu-O thin film tunnel junctions" (unpublished).

[90] P. M. Mankiewich et al., "Fabrication and characterization of an $\mathrm{YBa}_{2} \mathrm{Cu}_{3} \mathrm{O}_{7} / \mathrm{Au} / \mathrm{YBa}_{2} \mathrm{Cu}_{3} \mathrm{O}_{7} \mathrm{~S}-\mathrm{N}$-S microbridge," in Proc. 5th Int. Workshop on Future Electron Devices-High Temperature Superconducting Devices, pp. 157-160, 1988.

[91] R. H. Koch, W. J. Gallagher, B. Bumble, and W. Y. Lee, "Lownoise thin-film TI Ba Ca Cu O dc SQUIDs operated at $77 \mathrm{~K}$," Appl. Phys. Lett., vol. 54, pp. 951-953, 1989.

[92] R. H. Koch, "Performance related issues of SQUIDs made from high $T_{C}$ superconductors," Bull. Am. Phys. Soc., vol. 34, p. $573,1989$.

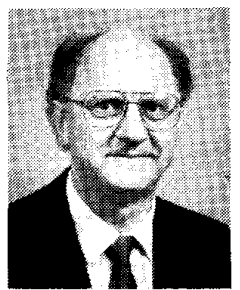

John Clarke was born in Cambridge, England, on February 10, 1942. He received the B.A. degree in 1964 and the M.A. and Ph.D. degrees in 1968, all from the University of Cambridge.

Since 1968 he has been with the Department of Physics at the University of California, Berkeley, and with the Materiais and Chemical Sciences Division of the Lawrence Berkeley Laboratory. His research has been mostly in fundamental properties of superconductors and in the development and applications of superconducting devices, notably superconducting quantum interference devices.

Dr. Clarke is a Fellow of the American Association for the Advancement of Science, the American Physical Society, and the Royal Society of London. He received the Charles Vernon Boys Prize of the British Institute of Physics in 1977 and the Fritz London Memorial Award in 1987. He was named California Scientist of the Year, also in 1987. 